\title{
Foliar Nutrition and Post-Harvest of Onion Seed: Effects of storage temperatures, storage period and foliar nutrition
}

\author{
Shehata, S. A. ${ }^{1}$, Hashem, M. Y. ${ }^{2}$, Mahmoud, G. I. ${ }^{3}$, Abd El-Gawad, K. F. ${ }^{1}$, El-Ramady, H. R.*4,5 \\ Alshaal, T. A. ${ }^{4,5}$, Domokos-Szabolcsy, É. ${ }^{5}$, Elhawat, N. ${ }^{5,6}$, Prokisch, J. ${ }^{7}$ and Fári, M. ${ }^{5}$ \\ ${ }^{1}$ Vegetable Crops Dept., Faculty of Agriculture, Cairo University, Egypt \\ ${ }^{2}$ Economic Entomology Dept., Faculty Agriculture, Cairo University, Egypt \\ ${ }^{3}$ Biochemistry Dept., Faculty of Agriculture, Cairo University, Egypt \\ ${ }^{4}$ Soil and Water Sciences Dept., Fac. of Agriculture, Kafrelsheikh Uni., Kafr El-Sheikh, Egypt \\ ${ }^{5}$ Plant Biotechnology Dept., Debrecen Uni., Böszörményi Útca. 138, 4032 Debrecen, Hungary \\ ${ }^{6}$ Biological and Environmental Sci. Dept., Faculty of Home Economics, Al-Azhar University, Egypt \\ ${ }^{7}$ Bio- and Environmental Enegetics Inst., Debrecen Uni., Böszörményi Ú. 138, 4032 Debrecen, Hungary
}

*Corresponding author: El-Ramady, H. (ramady2000@gmail.com)

\begin{abstract}
The aim of onion bulb storage is to meet consumer demand for extended availability of onions whilst maintaining product quality. The principal biological factors leading to onion bulb deterioration are respiration, resumption of growth and pathogen attack. In onion bulbs a dormant period, when sprouting and rooting cannot be induced, is followed by a period of internal changes that prepare the bulb for breaking of dormancy and subsequent growth. Out of storage, the bulb then proceeds towards flowering and seed production.

Two successive winter seasons of 2008/2009 and 2009/2010 were conducted under sandy soil conditions to study the effect of spraying with 12 commercial compounds on yield and yield components of onion seeds and storage The seed yield of each commercial compounds plot from previous experiment was divided into two groups, storage under room temperature and $5^{\circ} \mathrm{C}$. Seeds transferred immediately after drying to Increasing Export Competition of Some Vegetable Crops Project Laboratory located in Faculty of Agriculture, Cairo University. The effects of storage temperature, storage period and foliar with some commercial compounds on onion seed quality were considered. Storage in $5^{\circ} \mathrm{C}$ had the higher germination percentage than storage in room temperature. Results indicated that as storage period increased the germination percent decreased. The treatment with boron or amica in the first season had the highest germination percentage. While, the treatment with union $\mathrm{Zn}$, union feer, union $\mathrm{Mn}$, boron, elga 600, caboron, amica, hummer or amino X had the highest germination percentage in the second season. Storage in $5^{\circ} \mathrm{C}$ resulted in higher moisture content than storage in room temperature. Regarding the effect of storage period on moisture content, the water content was significantly increased with prolongation of storage period. The lowest values of water content were recorded for treatments with union feer, shams $\mathrm{K}$ or boron in the first season, and union feer, shams K, boron, magnesium, shetocare or hummer in the second one. Catalase activity was significantly decreased as storage period increased. The treatment with shams $\mathrm{K}$, boron, shetocare or amino X had the highest catalase activity in both seasons. Peroxidase activity was significantly decreased as storage period increased. Foliar application with boron had the highest peroxidase activity in both seasons. Seed stored in room temperature had the higher malondialdehyde content than those stored in $5^{\circ} \mathrm{C}$ in the second season. The malondialdehyde content increased as storage period increased. The treatment with magnesium, caboron and the control in the first season, and the treatment with magnesium and the control in the second season had the highest malondialdehyde content.
\end{abstract}

Keywords: Onion; pre-harvest; post-harvest, foliar application, seeds production, modified atmosphere packages

\section{Introduction}

The postharvest life of fruits and vegetables has been traditionally defined in terms of visual appearance (freshness, color, and absence of decay or physiological disorders) and texture (firmness, juiciness, and crispness). Although this concept involves aesthetic appeal and mechanical properties associated with quality, it disregards flavor and nutritional quality (Ayala-Zavala et al. 2004). Flavor plays an important role in consumer satisfaction and influences further consumption of fruits and foods in general (Pelayo et al. 2003). Fruits form an important part of our diet mainly as a source of energy, vitamins, minerals, and antioxidants. Postharvest losses in nutritional quality, particularly vitamin $\mathrm{C}$ content, can be substantial and are enhanced by physical damage, extended storage duration, high temperatures, low relative humidity, and chilling injury of chilling-sensitive commodities (Navarro et al. 2006; Kevers et al. 2007). 
Many biochemical characteristics change during storage of onion. These include changes in water content, the concentrations of flavor-related compounds (Uddin and MacTavish, 2003), organic acids, carbohydrates (Benkeblia et al. 2005), plant growth regulators and phenolics (Benkeblia, 2000). Biochemical changes during storage are likely to be linked with respiration. All nutrients required for growth of the sprout must come from within the bulb; therefore, changes in certain key characteristics might be used to predict the onset of sprouting. Maximal and minimal concentrations of certain substances are known to coincide with sprouting, but there is currently no biochemical assay that anticipates sprouting. During storage a gradual change in the relative composition of plant growth regulators occurs as the concentrations of growth promoters and/or growth inhibitors rise or fall, respectively. The growth inhibitory substance, detected by Thomas and Isenberg (1972), is widely believed to be abscisic acid (Chope et al. 2006).

The temperature at which onion sets are subjected in storage has a significant influence on bulb yield. Low storage temperature $\left(0{ }^{\circ} \mathrm{C}\right)$ leads to the highest bulb yield but this was reduced as the storage temperature was raised from 0 to $5{ }^{\circ} \mathrm{C}$. In general, high temperature storage (above 20 ${ }^{\circ} \mathrm{C}$ ) results in increased total bulb yields while very high temperature $\left(25.5-31{ }^{\circ} \mathrm{C}\right)$ or below $0{ }^{\circ} \mathrm{C}$ slightly depressed yield by delaying sprouting after planting the bulbs, thus shortening the period available for growth. On the other hand, storage of sets at mild temperatures $\left(5-15^{\circ} \mathrm{C}\right)$ usually results in lower marketable yields than do lower and higher storage temperatures (Khokhar et al. 2007). Many studies have stated that cold storage (about $0{ }^{\circ} \mathrm{C}$ ) prevents subsequent flowering and improves bulb yield, but leads to crops maturing earlier than those stored at high temperatures (about $25^{\circ} \mathrm{C}$ ) (Khokhar, 2009).

Accelerated ageing of seeds induced by several days of exposure to high temperature and humidity is recognized as an accurate indicator of seed viability and storability (Delouche and Baskin, 1973). Artificial accelerated ageing enables to understand the possible sequence of causes and reasons of seed deterioration at faster rate, thus helps in formulating counteractive priming treatments and appropriate storage practices. Rapidly deteriorating seeds show decline in their ability to emerge into vigorous seedlings (McDonald, 1999). Some of the deleterious effects of ageing are associated with damages occurring at membrane, nucleic acids and protein levels (Fujikura and Karssen, 1995). Peroxidation of unsaturated fatty acids is considered to be one of the main reasons for loss of storability, which occurs due to decreased levels of antioxidants, reduced activity of free radical and peroxide scavenging enzymes, and increased lipid peroxidation vis-à-vis malondialdehyde content (Bailly et al. 1996). Standardization of appropriate seed conditioning, packaging and storage conditions could ensure satisfactory planting quality of onion seeds at the time of sowing. In the present study, experiments were conducted to find out possible ways to improve storability of onion seeds with respect to packaging and storage conditions. Germination performance, activities of antioxidant enzymes and lipid peroxidation products were analyzed in rapidly aged onion seeds (Rao et al. 2006).

Onions (Allium cepa) are widely used around the world in food as seasoning with well known health benefits (Wood, 2008). However, onions are also reported to be susceptible to microbial contamination during postharvest storage (Calvin et al. 2004). Three microbial food-borne outbreaks in the USA, including four deaths and several illnesses, were associated with the consumption of imported onions of poor hygienic quality (Calvin et al. 2004). Along with hygienic concerns, sprouting is also a big problem in the long-terms to rage of onions (Ahn et al. 2012).

Onion is a species of the alliaceae family it is of great economic importance in Egypt. It is the most important cash crop after rice in Egypt. The total planted area for onion seed production is 2752 fed (ha $=2.4$ fed). Producing 742 tons with an average of $270 \mathrm{~kg} \mathrm{fed}^{-1}$ according to the Egyptian Ministry of Agriculture report (2008). Increasing its yield with consequent economic return is the major concern of the farmers (Abd El-Gawad, 2012). Egypt is considered the $4^{\text {th }}$ producer of dry onion in the world, where it produce about 2,208,080 ton, average yield per hectare is $358833 \mathrm{~kg} \mathrm{ha}^{-1}$ and the total harvested area from dry onion 61535 ha in 2010 (FAO, 2012).

The seed production programs depend upon quality of seeds, agronomic practices and plant protection measures taken to produce the healthy and vigorous crop. Among the agronomic practices nutrient management through organic sources is considered as an important factor for production. The problem of high cost of chemical fertilizers fully meet out nutrient requirement of crop by single source therefore integrated nutrients management such as organic matters like farmyard manure, vermicompost, poultry manure and biofertilizers use has become necessary (Bendegumbal, 2007).

Therefore, the aim of this study was to investigate the effect of foliar application with 12 commercial compounds on onion growth, seed production and its longevity. Also, the study aimed to investigate storage conditions such as storage temperature, storage period and foliar with some commercial compounds on onion seed quality.

\section{Materials and Methods}

\section{Experimental design}

The aim of this investigation was to study the effect of foliar application with 12 commercial compounds and untreated treatment (foliar with water) on onion seed production under sandy soil conditions (Fig. 1). These commercial compounds are listed in Table 1. The seeds for this study produced at Waddy Elnatron farm, Agricultural Experimental Station of the Faculty of Agriculture, Cairo University. This study conducted in two successive seasons in 2008/2009 and 2009/2010. Waddy Elnatron farm is reclaimed sand soil, its chemical and physical analysis is presented in Table 2 and water chemical analysis is presented in Table 3 . 
Table 1: Commercial compounds, their structures and using rate of them

\begin{tabular}{|c|c|c|c|}
\hline Compound & Company & Compound structure & Using rate \\
\hline Shetocare & Kemia Masr & $\begin{array}{l}1000 \mathrm{ppm} \mathrm{N}+500 \mathrm{ppm} \mathrm{K}+100 \mathrm{ppm} \mathrm{Zn}+50 \mathrm{ppm} \mathrm{Cu}+500 \mathrm{ppm} \mathrm{P}+100 \mathrm{ppm} \mathrm{Fe}+ \\
50 \mathrm{ppm} \mathrm{Mn}+50 \mathrm{ppm} \mathrm{B}\end{array}$ & $1 \mathrm{ml} \mathrm{l}^{-1}$ \\
\hline Amica & Technogreen & $10 \%$ amino acid $+5 \%$ total nitrogen $+14 \%$ calcium $+7 \%$ organic matter & $1 \mathrm{ml} \mathrm{l}^{-1}$ \\
\hline Caboron & Technogreen & $6 \%$ chelate calcium $+1.5 \%$ chelate $B+20 \%$ calcium oxide & $1 \mathrm{ml} \mathrm{l}^{-1}$ \\
\hline Amino $\mathrm{X}$ & UAD & $\begin{array}{l}80 \% \text { total amino acid }+16 \% \text { free amino acid }+10 \% \text { organic nitrogen }+2.5 \% \\
\text { potassium oxide }\end{array}$ & $\operatorname{lg~} 1^{-1}$ \\
\hline Elga 600 & Technogreen & $1 \% \mathrm{~N}+18 \%$ potassium oxide $+2 \% \mathrm{~S}$ & $1 \mathrm{~g}^{-1}$ \\
\hline Hummer & UAD & Humic acid containing $6 \%$ potassium oxide $+86 \%$ potassium hummat & $1 \mathrm{~g}^{-1}$ \\
\hline Union Zn & UAD & $12 \% \mathrm{Zn}$ chelated on amino and organic acids & $1 \mathrm{~g} \mathrm{l}^{-1}$ \\
\hline Union Mn & UAD & $13 \% \mathrm{Mn}$ chelated on amino and organic acids & $\operatorname{lg~1^{-1}}$ \\
\hline Union Feer & UAD & $6 \% \mathrm{Fe}$ chelated on amino and organic acids & $1 \mathrm{~g}^{-1}$ \\
\hline Shams K & UAD & $50 \%$ potassium oxide $+1 \%$ magnesium oxide & \\
\hline Boron & UAD & - & $0.5 \mathrm{~g} \mathrm{l}^{-1}$ \\
\hline Magnesium & UAD & - & $0.5 \mathrm{~g} \mathrm{l}^{-1}$ \\
\hline Untreated & & sprayed with water & - \\
\hline
\end{tabular}

* UAD = Union for Agricultural Development

Table 2: Physical and chemical analysis of the experimental soil in 2008/2009 and 2009/2010 seasons

\begin{tabular}{|c|c|c|c|c|c|c|c|c|}
\hline \multirow[t]{2}{*}{ Season } & \multirow[t]{2}{*}{ Soil texture } & \multicolumn{4}{|c|}{ Soluble cations (meq $\left.\mathbf{l}^{-1}\right)$} & \multicolumn{3}{|c|}{ Soluble anions $\left(\operatorname{meq} \mathrm{l}^{-1}\right)$} \\
\hline & & $\mathbf{K}^{+}$ & $\mathbf{N a}^{+}$ & $\mathbf{C a}^{++}$ & $\mathbf{M g}^{++}$ & $\mathrm{HCO}_{3}^{-}$ & $\mathrm{SO}_{4}^{+2}$ & $\mathrm{Cl}^{-}$ \\
\hline $2008 / 2009$ & Sandy & 10.0 & 17.52 & 16.11 & 6.37 & 1.33 & 21.67 & 18.0 \\
\hline $2009 / 2010$ & Sandy & 10.8 & 40.83 & 64.37 & 50.78 & 5.90 & 21.34 & 31.5 \\
\hline
\end{tabular}

Table 2: Cont.

\begin{tabular}{|c|c|c|c|c|c|c|c|c|}
\hline \multirow[t]{2}{*}{ Season } & \multirow[t]{2}{*}{ Soil EC $\left(\mathrm{dS} \mathrm{m}^{-1}\right)$} & \multirow[t]{2}{*}{ Soil pH } & \multicolumn{6}{|c|}{ Available nutrients $\left(\mathrm{mg} \mathrm{kg}^{-1}\right)$} \\
\hline & & & $\mathbf{N}$ & $\mathbf{P}$ & $\mathbf{F e}$ & $\mathbf{C u}$ & $\mathbf{Z n}$ & Mn \\
\hline $2008 / 2009$ & 4.20 & 7.70 & 18.0 & 28.2 & 17.5 & 1.20 & 2.40 & 5.20 \\
\hline $2009 / 2010$ & 3.90 & 8.35 & 18.9 & 35.4 & 16.2 & 1.55 & 3.18 & 6.28 \\
\hline
\end{tabular}

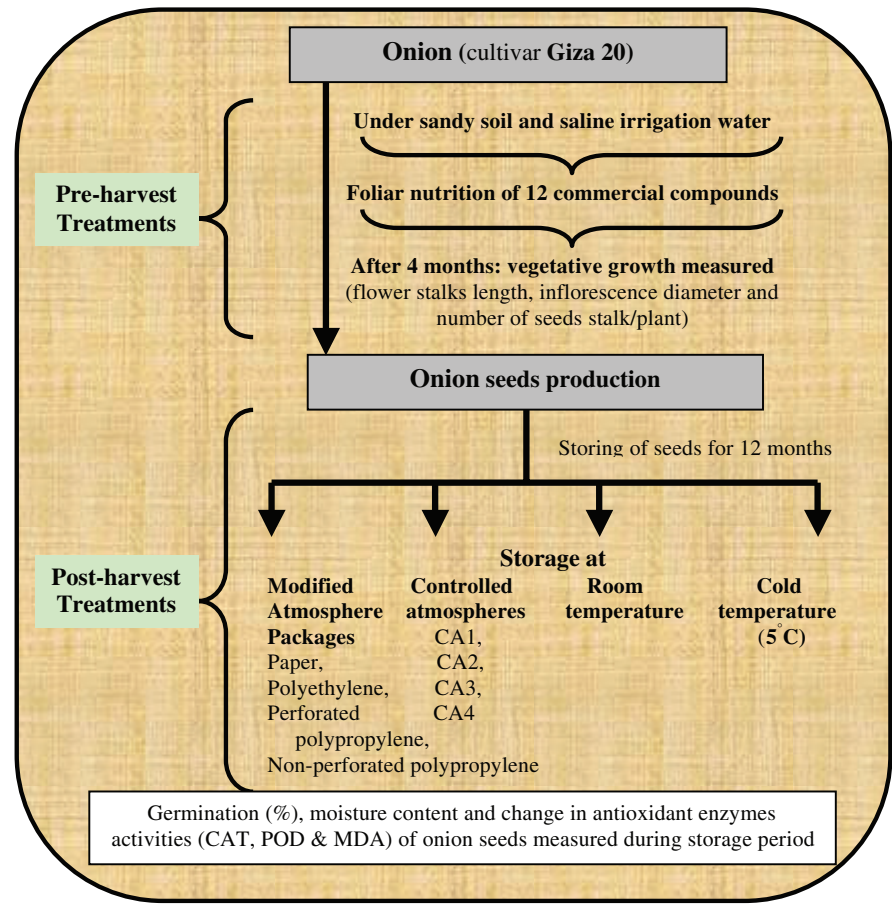

Figure 1: Experimental design
One commercial onion cultivar was selected for this study, viz., Giza 20. Bulbs were brought from Agricultural Research Center, Giza. Bulbs were sown on Dec. $18^{\text {th }}$ and $14^{\text {th }}$ in 2008 and 2009, respectively. Seeds were harvested on June, 14 and 7 in 2009 and 2010, respectively when the open capsules reached to $5 \%$ of total capsules. The area of each plot was $16 \mathrm{~m}^{2}$ (4 rows $\times 4 \mathrm{~m}$ long $\times 1 \mathrm{~m}$ width). Bulbs were sown on the center of the ridge and spaced 25 $\mathrm{cm}$ between each bulb in four replicates using standard commercial practices. The method of irrigation was drip. A completely randomize blocks design (CRBD) was used with four replicates. The commercial compounds were treated three times during the season of growth at vegetative growth, flowering and flower set (after two months of sowing date, one month later and one month later, respectively).

\section{Biochemical assessments of onion seed}

Two grams of seeds were ground in a mortar and homogenized in $20 \mathrm{ml}$ of $0.1 \mathrm{M}$ phosphate puffer $(\mathrm{pH} 7.8$ ) containing $0.4 \mathrm{~g}$ polyvinyl pyrrolidone, $2 \mathrm{mM}$ dithiotheitol and $0.1 \mathrm{mM}$ EDTA followed by centrifuging at $16,000 \times \mathrm{g}$ for $15 \mathrm{~min}$ at $4^{\circ} \mathrm{C}$ (Rao et al. 2006). 
Table 3: Chemical analysis of irrigation water

\begin{tabular}{|c|c|c|c|c|c|c|c|c|}
\hline \multirow{2}{*}{$\begin{array}{l}\text { Water EC (dS } \\
\left.\mathbf{m}^{-1}\right)\end{array}$} & \multirow[t]{2}{*}{ Water pH } & \multicolumn{4}{|c|}{ Soluble cations (meq $\left.\mathrm{l}^{-1}\right)$} & \multicolumn{3}{|c|}{ Soluble anions (meq $\mathrm{I}^{-1}$ ) } \\
\hline & & $\mathbf{K}^{+}$ & $\mathbf{N a}^{+}$ & $\mathbf{C a}^{++}$ & $\mathbf{M g}^{++}$ & $\mathrm{CO}_{3}^{--}$ & $\mathrm{SO}_{4}^{+2}$ & $\mathrm{Cl}^{-}$ \\
\hline 3.9 & 7.5 & 0.39 & 29.88 & 2.16 & 7.15 & 4.04 & 12.71 & 22.83 \\
\hline
\end{tabular}

Table 4: Some vegetables, their storage conditions, and material used for analysis (adapted from Kevers et al. 2007)

\begin{tabular}{|c|c|c|c|}
\hline \multirow[t]{2}{*}{ Vegetable } & \multicolumn{2}{|c|}{ Storage } & \multirow[t]{2}{*}{ Material used } \\
\hline & Condition & Duration (days) & \\
\hline Asparagus & $4{ }^{\circ} \mathrm{C}$ & 22 & All material \\
\hline Broccoli & $4{ }^{\circ} \mathrm{C}$ Packaged with polypropylene films & 27 & All material \\
\hline Carrot & $4{ }^{\circ} \mathrm{C}$ & 51 & Without peel \\
\hline Celery & $4{ }^{\circ} \mathrm{C}$ & 8 & Without peel \\
\hline Cherry & Room temperature & 7 & Without hard core \\
\hline Cucumber & $4^{\circ} \mathrm{C}$ & 8 & With skin \\
\hline French bean & $4^{\circ} \mathrm{C}$ & 8 & All material \\
\hline Garlic & Room temperature & 30 & Without peels \\
\hline Green pepper & $4{ }^{\circ} \mathrm{C}$ & 14 & Without pips \\
\hline Lettuce & $4{ }^{\circ} \mathrm{C}$ Packaged in sealed polypropylene bags & 8 & All material \\
\hline Onion & Room temperature & 23 & Without peel \\
\hline Red pepper & $4{ }^{\circ} \mathrm{C}$ & 14 & Without pips \\
\hline Spinach & $4{ }^{\circ} \mathrm{C}$ Packaged in sealed polypropylene bags & 19 & All material \\
\hline Tomato & $4{ }^{\circ} \mathrm{C}$ & 36 & All fruit \\
\hline Yellow pepper & $4^{\circ} \mathrm{C}$ & 34 & Without pips \\
\hline
\end{tabular}

\section{Catalase activity (CAT)}

Catalase was assayed by measuring the decrease in absorbance due to disappearance of $\mathrm{H}_{2} \mathrm{O}_{2}$ at $240 \mathrm{~nm}$ according to Chance and Maehly (1955). The enzyme extract (100 $\mu \mathrm{l})$ was added to $100 \mu \mathrm{l}$ of $100 \mathrm{mM} \mathrm{H} \mathrm{O}_{2}$ and the total volume was made up to $1 \mathrm{ml}$ by $250 \mathrm{mM}$ phosphate buffer $\mathrm{pH} 6.8$. The decreasing in optical density at $240 \mathrm{~nm}$ against blank was recovered every minute. For reproducible results, the absorbance at $240 \mathrm{~nm}$ should be between $0.450-0.500$ and start decrease by adding enzyme extract.

\section{Peroxidase activity (POD)}

This enzyme assayed spectrophotochemically according to Amako et al. (1994). The assay was carried out at $25^{\circ} \mathrm{C}$ in $1.0 \mathrm{~cm}$ light bath cuvette and the reaction mixture was consisted of $1500 \mu \mathrm{l}$ phosphate buffer, 1000 $\mu \mathrm{l}$ pyrogallol and $480 \mu \mathrm{l} \mathrm{H}_{2} \mathrm{O}_{2}$ solution. After mixing, the reaction was initiated by adding the enzyme extract $(20$ $\mu \mathrm{l})$ and increasing in optical density at $430 \mathrm{~nm}$ against blank (without extract) was continuously recorded every minute for three minutes. The calculation was per enzyme unit (EU), where this unit definition (EU) is defined as the amount of enzyme required to cause an increase in the optical density at $430 \mathrm{~nm} / \mathrm{min}$ at $25^{\circ} \mathrm{C}$ under standard conditions $(0.01 \mathrm{OD}=1 \mathrm{EU})$.

\section{Malondialdehyde content (MDA)}

Seed material $(0.5 \mathrm{~g})$ was homogenized in $5 \mathrm{ml}$ of $0.1 \%$ trichloroacetic acid (TCA) and centrifuged at $20,000 \times \mathrm{g}$ for $10 \mathrm{~min}$ to $1 \mathrm{ml}$ of extract, $4 \mathrm{ml}$ of $0.5 \%$ (w/v) thiobarbituric acid (TBA) in $20 \%(\mathrm{w} / \mathrm{v})$ TCA was added. The homogenate was incubated at $95^{\circ} \mathrm{C}$ for $30 \mathrm{~min}$. cooled on ice and centrifuged at $16,000 \times \mathrm{g}$ for $30 \mathrm{~min}$ and MDA content $\left(\mathrm{mmol} \mathrm{g}{ }^{-1} \mathrm{fw}\right.$ ) was spectrometrically determined at $452 \mathrm{~nm}$ according to Health and Parker (1968).

\section{Effect of storage temperatures, storage periods and foliar application with some commercial compounds on onion seeds quality}

This part of the investigation aimed to study the effect of different commercial compounds and two storage temperatures (room temperature and $5^{\circ} \mathrm{C}$ ) on germination, moisture content and change in antioxidant enzymes activity of onion seeds during storage period. The seeds were transferred immediately after drying to Increasing Export Competition of Some Vegetable Crops Project laboratory located in Faculty of Agriculture, Cairo University. Seed yield of each commercial compound plot was divided into two groups, storage under room temperature and $5^{\circ} \mathrm{C}$. Seeds were stored for one year. Number of treatments was 104 (12 commercial compounds + control $\times 2$ storage temperatures $\times 4$ replicates). All treatments were stored in non perforated polypropylene bags. The following parameters were measured after harvest and every 4 months: seed water content, seed germination, chemical analysis and biochemical assessment (CAT, and POD and MDA content).

\section{Statistical analysis}

Data were organized in a completely randomized block design (CRBD). Analyses of variance (ANOVA) were obtained using M. State statistical software. The new LSD 
Table 5: Antioxidant Capacity (DPPH and ORAC) and vegetable content in total phenolics, ascorbic acid, and total flavonoids* (adapted from Kevers et al. 2007)

\begin{tabular}{|c|c|c|c|c|c|}
\hline Vegetable & $\begin{array}{c}\text { Total phenolics } \\
\text { (mg of CAE } 100 \\
\mathrm{~g}^{-1} \text { of FW) } \\
\end{array}$ & $\begin{array}{c}\text { DPPH } \\
(\mu \mathrm{M} \text { TE } 100 \\
\left.\mathrm{g}^{-1} \text { of } \mathrm{FW}\right) \\
\end{array}$ & $\begin{array}{c}\text { ORAC } \\
(\mu \mathrm{M} \text { TE } 100 \\
\left.\mathrm{g}^{-1} \text { of } \mathrm{FW}\right) \\
\end{array}$ & $\begin{array}{c}\text { Ascorbic acid } \\
\text { (mg of AA } 100 \\
\mathrm{~g}^{-1} \text { of FW) }\end{array}$ & $\begin{array}{c}\text { Total flavonoids } \\
\text { (mg of QE } 100 \\
\mathrm{~g}^{-1} \text { of FW) }\end{array}$ \\
\hline Red pepper & $296 \pm 13$ & $1207 \pm 48$ & $875 \pm 152$ & $165.6 \pm 15.8$ & $4.8 \pm 0.5$ \\
\hline Yellow pepper & $284 \pm 10$ & $1207 \pm 124$ & $1011 \pm 120$ & $171.3 \pm 18.9$ & $2.3 \pm 0.1$ \\
\hline Green pepper & $215 \pm 31$ & $1163 \pm 104$ & $907 \pm 196$ & $135.3 \pm 12.9$ & $2.1 \pm 0.4$ \\
\hline Spinach & $177 \pm 2$ & $184 \pm 4$ & $1558 \pm 64$ & $12.4 \pm 0.5$ & $6.6 \pm 0.5$ \\
\hline Broccoli & $127 \pm 32$ & $188 \pm 8$ & $1586 \pm 328$ & $15.2 \pm 4.2$ & $0.3 \pm 00$ \\
\hline Garlic & $113 \pm 4$ & $60 \pm 4$ & $1370 \pm 392$ & $4.9 \pm 0.7$ & $0.2 \pm 0$ \\
\hline Leek & $77 \pm 3$ & $180 \pm 4$ & $675 \pm 44$ & $21.9 \pm 1.2$ & $0.4 \pm 01$ \\
\hline Celery & $75 \pm 4$ & $60 \pm 4$ & $679 \pm 68$ & $0.5 \pm 0.1$ & $0.2 \pm 0.1$ \\
\hline Onion & $53 \pm 7$ & $60 \pm 4$ & $739 \pm 144$ & $7.0 \pm 0.7$ & $3.3 \pm 0.4$ \\
\hline Asparagus & $44 \pm 4$ & $72 \pm 4$ & $296 \pm 68$ & $9.2 \pm 0.5$ & $0.2 \pm 0.1$ \\
\hline Tomato & $35 \pm 2$ & $84 \pm 4$ & $216 \pm 4$ & $8.2 \pm 1.2$ & $2.3 \pm 0.2$ \\
\hline French bean & $34 \pm 1$ & $68 \pm 4$ & $511 \pm 88$ & $0.5 \pm 0$ & $5.0 \pm 0.2$ \\
\hline Lettuce & $32 \pm 1$ & $56 \pm 4$ & $184 \pm 12$ & $0 \pm 0$ & $2.6 \pm 0.3$ \\
\hline Cucumber & $20 \pm 2$ & $0 \pm 0$ & $160 \pm 32$ & $0.4 \pm 0.3$ & $0.2 \pm 0.0$ \\
\hline Carrot & $0 \pm 0$ & $0 \pm 0$ & $276 \pm 100$ & $1.6 \pm 0.2$ & $0.7 \pm 0.1$ \\
\hline
\end{tabular}

* Assays were run immediately after vegetables were obtained from distribution center

Abbreviation: DPPH = 2,2-diphenyl-1-picrylhydrazyl ORAC $=$ oxygen radical absorbance capacity $\mathrm{TE}=$ Trolox equivalents $\mathrm{QE}=$ quercetin equivalents

$\mathrm{CAE}=$ chlorogenic acid equivalents

method (Waller and Duncan, 1969) was used for testing the significance of means in all experiments conducted.

\section{Results and Discussions}

\section{Storage of onion seed under cold temperature}

Storage of fresh fruits and vegetables prolongs their usefulness. The principal goal of storage is to preserve the commodity in its most useable form for the consumer. The extension of storage life and the improvement of quality of fresh fruits and vegetables can be supplied by harvesting at proper maturity, control of post-harvest diseases, chemical treatments, refrigeration, controlled and modified atmospheres. The main goals of storage are to (1) slow the biological activity of fruits and vegetables (2) slow the growth of microorganisms (3) reduce transpirational losses. In general, fruits and vegetables visually spoil before any significant antioxidant capacity loss occurs. Nevertheless, it could be stressed that, in general, polyphenolic content increased. Increased levels of antioxidant capacity generally accompanied this increase, which should be considered as an important assurance for the impact of storage evolution of phenolics on the nutritional value of fruits and vegetables (Tables 4 and 5).

\section{Germination percent}

\section{Effect of storage temperature on germination percent}

Storage temperature had a significant effect on germination percent of onion seeds in both seasons. Storage at cold temperature had the higher germination percent than storage at room temperature (Table 6). These results are in agreement with those obtained by Garg and Pitam (2005) and Lazarenko and Bezrukov (2008) as they found that the germination percentage decreased as storage temperature increased.

\section{Effect of storage periods on germination percent}

Regarding the effect of storage period on germination percentage, data in Table $\mathbf{6}$ show that germination percentage decreased as storage period increased. These results are in agreement with those obtained by Muhammad and Anjum (2002), Sharma et al. (2002 and 2004) and Gaviola et al. (2006). These results may be attributed to the decrease of antioxidant enzyme activity as storage period increased and the increasing of water content and malondialdehyde content as storage period increased.

\section{Effect of foliar application with commercial compounds on germination percent}

Concerning the effect of foliar application with commercial compounds on germination percentage, data in Table 6 show that the treatment with boron or amica in the first season had the highest germination percentage. While, the treatments with union $\mathrm{Zn}$, union feer, union $\mathrm{Mn}$, boron, elga 600, caboron, amica, hummer or amino $\mathrm{X}$ had the highest germination percentage in the second season. These results may be due to the fact that these treatments had the highest values of enzymes activity and the lowest malondialdehyde content. These results are in agreement with Xin and Wang (2006) and Demirkaya et al. (2010). They found highly positive correlation between the loss of seed viability and the decreases that occurred in catalase (CAT), peroxidase (POD) and superoxide dismutase 
Table 6: Effect of storage temperature, storage period and foliar application with commercial compounds on germination (\%) and water content (\%) of onion seeds during seasons of 2008 / 2009 and 2009 / 2010

\begin{tabular}{|c|c|c|c|c|}
\hline \multirow[t]{2}{*}{ Treatments } & \multicolumn{2}{|c|}{ Germination (\%) } & \multicolumn{2}{|c|}{ Water content $(\%)$} \\
\hline & $2008 / 2009$ & $2009 / 2010$ & $2008 / 2009$ & $2009 / 2010$ \\
\hline $\begin{array}{l}\text { Storage temperature } \\
\text { Room temperature } \\
\text { Cold temperature }\left(5^{\circ} \mathrm{C}\right) \\
\text { LSD }_{0.05}\end{array}$ & $\begin{array}{c}81.32 \mathrm{~b} \\
85.66 \mathrm{a} \\
2.784\end{array}$ & $\begin{array}{c}83.38 \mathrm{~b} \\
86.83 \mathrm{a} \\
1.260\end{array}$ & $\begin{array}{c}4.145 \mathrm{~b} \\
5.191 \mathrm{a} \\
0.013\end{array}$ & $\begin{array}{c}4.586 \mathrm{a} \\
4.572 \mathrm{a} \\
\text { N.S. }\end{array}$ \\
\hline $\begin{array}{c}\text { Storage period (month) } \\
0 \\
4 \\
8 \\
12 \\
\text { LSD }_{\mathbf{0 . 0 5}} \\
\end{array}$ & $\begin{array}{l}94.46 \mathrm{a} \\
86.62 \mathrm{~b} \\
78.93 \mathrm{c} \\
73.96 \mathrm{~d} \\
0.6131\end{array}$ & $\begin{array}{l}94.10 \mathrm{a} \\
90.04 \mathrm{~b} \\
81.39 \mathrm{c} \\
74.90 \mathrm{~d} \\
1.008\end{array}$ & $\begin{array}{l}4.179 \mathrm{~d} \\
4.473 \mathrm{c} \\
5.272 \mathrm{a} \\
4.747 \\
\text { b } 0.068\end{array}$ & $\begin{array}{l}4.200 \mathrm{~d} \\
4.960 \mathrm{a} \\
4.666 \mathrm{~b} \\
4.490 \mathrm{c} \\
0.0441 \\
\end{array}$ \\
\hline $\begin{array}{c}\text { Foliar application } \\
\text { Union } \mathrm{Zn} \\
\text { Union Feer } \\
\text { Union } \mathrm{Mn} \\
\text { Shams K } \\
\text { Boron } \\
\text { Magnesium } \\
\text { Elga } 600 \\
\text { Caboron } \\
\text { Amica } \\
\text { Shetocare } \\
\text { Hummer } \\
\text { Amino X } \\
\text { Untreated } \\
\text { LSD }_{0.05} \\
\end{array}$ & $\begin{array}{l}84.72 \mathrm{c}-\mathrm{d} \\
85.19 \mathrm{~b}-\mathrm{c} \\
84.63 \mathrm{c}-\mathrm{d} \\
81.63 \mathrm{e}-\mathrm{f} \\
87.41 \mathrm{a} \\
79.09 \mathrm{~g} \\
82.78 \mathrm{~d}-\mathrm{e} \\
84.16 \mathrm{c}-\mathrm{d} \\
86.84 \mathrm{a}-\mathrm{b} \\
80.28 \mathrm{f}-\mathrm{g} \\
83.28 \mathrm{c}-\mathrm{e} \\
84.84 \mathrm{~b}-\mathrm{d} \\
80.56 \mathrm{f}-\mathrm{g} \\
2.093\end{array}$ & $\begin{array}{c}85.19 \text { a-c } \\
87.00 \text { a } \\
86.25 \text { a-b } \\
84.28 \text { b-d } \\
86.69 \text { a } \\
81.53 \text { e } \\
84.84 \text { a-d } \\
86.41 \text { a-b } \\
86.09 \text { a-b } \\
83.59 \text { c-e } \\
85.44 \text { a-c } \\
86.31 \text { a-b } \\
82.78 \text { d-e } \\
2.235\end{array}$ & $\begin{array}{c}4.62 \mathrm{~b}-\mathrm{e} \\
4.59 \mathrm{~d}-\mathrm{e} \\
4.68 \mathrm{a}-\mathrm{e} \\
4.60 \mathrm{c}-\mathrm{e} \\
4.57 \mathrm{e} \\
4.66 \mathrm{a}-\mathrm{e} \\
4.67 \mathrm{a}-\mathrm{e} \\
4.64 \mathrm{a}-\mathrm{e} \\
4.74 \mathrm{a} \\
4.72 \mathrm{a}-\mathrm{c} \\
4.72 \mathrm{a}-\mathrm{b} \\
4.69 \mathrm{a}-\mathrm{d} \\
4.73 \mathrm{a}-\mathrm{b} \\
0.114\end{array}$ & $\begin{array}{l}4.65 \mathrm{a}-\mathrm{b} \\
4.57 \mathrm{c}-\mathrm{e} \\
4.61 \mathrm{~b}-\mathrm{d} \\
4.50 \mathrm{e}-\mathrm{g} \\
4.51 \mathrm{e}-\mathrm{f} \\
4.44 \mathrm{~g} \\
4.60 \mathrm{~b}-\mathrm{d} \\
4.65 \mathrm{a}-\mathrm{b} \\
4.68 \mathrm{a} \\
4.45 \mathrm{f}-\mathrm{g} \\
4.54 \mathrm{~d}-\mathrm{e} \\
4.62 \mathrm{a}-\mathrm{c} \\
4.64 \mathrm{a}-\mathrm{c} \\
0.074\end{array}$ \\
\hline
\end{tabular}

Details of treatment listed in Table 3

Table 7: Effect of the interaction between storage temperature and storage period on germination percentage of onion seeds during seasons of 2008/2009 and $2009 / 2010$

\begin{tabular}{|c|c|c|c|c|}
\hline \multirow{3}{*}{$\begin{array}{l}\text { Storage period } \\
\text { (month) }\end{array}$} & \multicolumn{4}{|c|}{ Storage temperature } \\
\hline & Room temperature & Cold temp. $\left(5^{\circ} \mathrm{C}\right)$ & Room temperature & Cold temp. $\left(5^{\circ} \mathrm{C}\right)$ \\
\hline & \multicolumn{2}{|c|}{ Season $2008 / 2009$} & \multicolumn{2}{|c|}{ Season $2009 / 2010$} \\
\hline $\mathbf{0}$ & $94.48 \mathrm{a}$ & 94.48 a & $94.0 \mathrm{a}$ & $94.0 \mathrm{a}$ \\
\hline 4 & $84.77 \mathrm{c}$ & $88.46 \mathrm{~b}$ & $88.4 \mathrm{c}$ & $91.65 \mathrm{~b}$ \\
\hline 8 & $75.37 \mathrm{f}$ & $82.50 \mathrm{~d}$ & $79.34 \mathrm{e}$ & $83.44 \mathrm{~d}$ \\
\hline 12 & $70.67 \mathrm{~g}$ & $77.25 \mathrm{e}$ & $71.67 \mathrm{f}$ & $78.13 \mathrm{e}$ \\
\hline $\mathbf{L S D}_{0.05}$ & 0.8671 & & 1.425 & \\
\hline
\end{tabular}

Table 8: Effect of the interaction between storage temperature and foliar application with commercial compounds on germination percentage of onion seeds during seasons of 2008/2009 and 2009/2010

\begin{tabular}{|c|c|c|c|c|}
\hline \multirow{3}{*}{ Treatments } & \multicolumn{4}{|c|}{ Storage temperature } \\
\hline & Room temperature & Cold temp. $\left(5^{\circ} \mathrm{C}\right)$ & Room temperature & Cold temp. $\left(5^{\circ} \mathrm{C}\right)$ \\
\hline & \multicolumn{2}{|c|}{ Season $2008 / 2009$} & \multicolumn{2}{|c|}{ Season 2009/2010 } \\
\hline Union Zn & $82.9 \mathrm{f}-\mathrm{h}$ & $86.5 \mathrm{~b}-\mathrm{d}$ & $83.63 \mathrm{f}-\mathrm{i}$ & 86.75 a-f \\
\hline Union Feer & $84.9 \mathrm{c}-\mathrm{h}$ & $85.4 \mathrm{c}-\mathrm{f}$ & 84.63 d-h & 89.38 a \\
\hline Union Mn & $82.1 \mathrm{~h}-\mathrm{j}$ & 87.1 b-c & 85.69 b-g & 86.81 a-e \\
\hline Shams K & $79.3 \mathrm{k}-1$ & $86.5 \mathrm{~b}-\mathrm{c}$ & 84.06 e-h & 84.50 d-h \\
\hline Boron & 83.0 e-i & $91.7 \mathrm{a}$ & $85.38 \mathrm{~b}-\mathrm{g}$ & 88.00 a-c \\
\hline Magnesium & 74.31 & $83.8 \mathrm{~d}-\mathrm{i}$ & $79.56 \mathrm{j}$ & $83.50 \mathrm{~g}-\mathrm{i}$ \\
\hline Elga 600 & $79.6 \mathrm{j}-\mathrm{k}$ & $85.8 \mathrm{c}-\mathrm{f}$ & $80.75 \mathrm{i}-\mathrm{j}$ & 88.94 a \\
\hline Caboron & $83.8 \mathrm{~d}-\mathrm{i}$ & $84.4 \mathrm{c}-\mathrm{h}$ & 84.63 d-h & 88.19 a-b \\
\hline Amica & $84.3 \mathrm{c}-\mathrm{h}$ & $89.3 \mathrm{a}-\mathrm{b}$ & 83.88 e-i & $88.31 \mathrm{a}-\mathrm{b}$ \\
\hline Shetocare & $78.1 \mathrm{k}$ & $82.3 \mathrm{~g}-\mathrm{j}$ & $82.25 \mathrm{~h}-\mathrm{j}$ & $84.94 \mathrm{c}-\mathrm{h}$ \\
\hline Hummer & $85.1 \mathrm{c}-\mathrm{g}$ & $81.3 \mathrm{i}-\mathrm{j}$ & 84.00 e-h & 86.88 a-e \\
\hline Amino $X$ & $83.6 \mathrm{~d}-\mathrm{i}$ & $86.0 \mathrm{c}-\mathrm{e}$ & $85.38 \mathrm{~b}-\mathrm{g}$ & $87.25 \mathrm{a}-\mathrm{d}$ \\
\hline Untreated & 78.0 & $83.1 \mathrm{e}-\mathrm{i}$ & $80.19 \mathrm{j}$ & 85.38 b-h \\
\hline LSD $_{0.05}$ & \multicolumn{2}{|c|}{2.960} & \multicolumn{2}{|c|}{3.161} \\
\hline
\end{tabular}

Details of treatment listed in Table 3 
Table 9: Effect of the interaction between storage period and foliar application on germination percentage of onion seeds during 2008 / 2009 and 2009/2010 seasons

\begin{tabular}{|c|c|c|c|c|c|c|c|c|}
\hline \multirow[t]{3}{*}{ Treatments } & \multicolumn{8}{|c|}{ Storage period (month) } \\
\hline & $\mathbf{0}$ & 4 & 8 & 12 & $\mathbf{0}$ & 4 & 8 & 12 \\
\hline & \multicolumn{4}{|c|}{ Season 2008/2009 } & \multicolumn{4}{|c|}{ Season $2009 / 2010$} \\
\hline Union $\mathrm{Zn}$ & 93.7 b-f & $89.1 \mathrm{~g}-\mathrm{k}$ & 80.5 o-r & $75.5 \mathrm{t}-\mathrm{x}$ & $93.0 \mathrm{a}-\mathrm{e}$ & $88.8 \mathrm{f}-\mathrm{i}$ & $81.2 \mathrm{k}-\mathrm{o}$ & $77.6 \mathrm{n}-\mathrm{q}$ \\
\hline Union Feer & $96.5 \mathrm{a}-\mathrm{b}$ & 87.7 h-1 & 80.7 o-r & 75.7 s-w & $95.5 a-b$ & $91.2 \mathrm{~b}-\mathrm{g}$ & 85.0 i-k & $76.2 \mathrm{p}-\mathrm{q}$ \\
\hline Union Mn & $95.2 \mathrm{~b}-\mathrm{d}$ & 87.6 h-1 & 80.1 o-r & $75.5 \mathrm{t}-\mathrm{x}$ & $94.7 \mathrm{a}-\mathrm{c}$ & $90.1 \mathrm{~d}-\mathrm{h}$ & $81.2 \mathrm{k}-\mathrm{o}$ & $78.8 \mathrm{~m}-\mathrm{p}$ \\
\hline Shams K & $91.5 \mathrm{~d}-\mathrm{h}$ & $86.0 \mathrm{j}-\mathrm{n}$ & 76.7 r-1 & $72.2 \mathrm{X}-\mathrm{Z}$ & $95.5 \mathrm{a}-\mathrm{b}$ & $91.1 \mathrm{~b}-\mathrm{g}$ & $79.51-\mathrm{p}$ & $71.0 \mathrm{r}$ \\
\hline Boron & $99.5 \mathrm{a}$ & 88.6 g-k & $83.5 \mathrm{~m}-\mathrm{p}$ & $78.0 \mathrm{r}-\mathrm{u}$ & 96.7 a & $91.1 \mathrm{~b}-\mathrm{g}$ & $81.6 \mathrm{k}-\mathrm{o}$ & $77.2 \mathrm{n}-\mathrm{q}$ \\
\hline Magnesium & $91.0 \mathrm{e}-\mathrm{i}$ & $83.5 \mathrm{~m}-\mathrm{p}$ & $73.5 \mathrm{w}-\mathrm{x}$ & $68.3 \mathrm{z}$ & 92.5 a-e & $87.7 \mathrm{~h}-\mathrm{j}$ & $76.51-\mathrm{p}$ & $69.3 \mathrm{r}$ \\
\hline Elga 600 & $94.5 \mathrm{~b}-\mathrm{e}$ & 86.1 j-n & $77.7 \mathrm{r}-\mathrm{v}$ & 72.7 w-y & $94.2 \mathrm{a}-\mathrm{d}$ & $89.1 \mathrm{e}-\mathrm{i}$ & $82.7 \mathrm{k}-\mathrm{n}$ & 73.2 q-r \\
\hline Caboron & $96.0 \mathrm{a}-\mathrm{c}$ & $85.8 \mathrm{k}-\mathrm{n}$ & $79.8 \mathrm{p}-\mathrm{s}$ & $74.8 \mathrm{u}-\mathrm{x}$ & $94.2 \mathrm{a}-\mathrm{d}$ & $92.2 \mathrm{~b}-\mathrm{g}$ & $81.5 \mathrm{o}-\mathrm{q}$ & $77.6 \mathrm{n}-\mathrm{q}$ \\
\hline Amica & 93.7 b-f & $90.1 \mathrm{f}-\mathrm{j}$ & $84.21-0$ & $79.2 \mathrm{q}-\mathrm{t}$ & $93.5 \mathrm{a}-\mathrm{e}$ & $90.1 \mathrm{~d}-\mathrm{h}$ & $83.6 \mathrm{k}-\mathrm{m}$ & $77.1 \mathrm{o}-\mathrm{q}$ \\
\hline Shetocare & $95.5 \mathrm{a}-\mathrm{d}$ & $83.1 \mathrm{n}-\mathrm{q}$ & $73.7 \mathrm{v}-\mathrm{X}$ & $68.7 \mathrm{y}-\mathrm{z}$ & $94.2 \mathrm{a}-\mathrm{d}$ & $90.3 \mathrm{c}-\mathrm{h}$ & $79.31-p$ & $70.3 \mathrm{r}$ \\
\hline Hummer & $92.5 \mathrm{~b}-\mathrm{f}$ & $87.2 \mathrm{i}-\mathrm{m}$ & $79.1 \mathrm{q}-\mathrm{t}$ & $74.2 \mathrm{u}-\mathrm{x}$ & 93.0 a-e & $89.7 \mathrm{e}-\mathrm{h}$ & $82.8 \mathrm{k}-\mathrm{m}$ & $76.1 \mathrm{p}-\mathrm{q}$ \\
\hline Amino $X$ & $96.2 \mathrm{a}-\mathrm{b}$ & 87.6 h-m & 80.8 o-r & $74.6 u-x$ & $93.2 \mathrm{a}-\mathrm{e}$ & $90.7 \mathrm{c}-\mathrm{h}$ & $83.6 \mathrm{j}-1$ & $77.6 \mathrm{n}-\mathrm{q}$ \\
\hline Untreated & $92.0 \mathrm{c}-\mathrm{g}$ & $83.2 \mathrm{n}-\mathrm{q}$ & $75.3 \mathrm{t}-\mathrm{w}$ & $71.6 \mathrm{x}-\mathrm{z}$ & 92.7 a-e & $87.8 \mathrm{~g}-\mathrm{j}$ & $79.21-\mathrm{p}$ & $71.2 \mathrm{r}$ \\
\hline $\mathbf{L S D}_{0.05}$ & \multicolumn{4}{|l|}{4.186} & \multicolumn{4}{|l|}{4.470} \\
\hline
\end{tabular}

Details of treatment listed in Table 3

(SOD) activity in the seeds and negative correlation between the loss of seed viability and the increases that occurred in malondialdehyde content in the seeds.

\section{Effect of the interaction between storage temperature and storage period}

Regarding the effect of the interaction between storage temperatures and storage period, the highest germination percentage after 12 months of storage was recorded at cold storage temperature $\left(5^{\circ} \mathrm{C}\right)$. The germination percentage was decreased by increasing storage period in the two storage temperatures, but the germination percentage still higher in cold storage temperature than in room temperature at all storage periods in both seasons (Table 7).

\section{Effect of the interaction between storage temperature and foliar application with commercial compounds}

Concerning the effect of the interaction between storage temperature and foliar application with commercial compounds, the highest germination percentage was recorded for the treatment with union $\mathrm{Zn}$, union $\mathrm{Mn}$, shams $\mathrm{k}$, boron, amica or amino $\mathrm{X}$ at $5^{\circ} \mathrm{C}$ in the first season and the treatment with union feer or elga 60 at $5^{\circ} \mathrm{C}$ in the second season as shown in Table 8.

\section{Effect of the interaction between storage period and foliar application with commercial compounds}

Data in Table 9 show that germination percentage decreased as storage period increased in all foliar treatments. The highest germination percentage at the end of storage period was recorded for the treatment with boron or amica in the first season and the treatment with union $\mathrm{Zn}$, union $\mathrm{Mn}$, boron, caboron or amino $\mathrm{X}$ in the second season. These results may be due to that these treatments had the highest germination percentage after harvest (Table 7).

\section{Effect of the interaction between storage temperatures, storage period and foliar application with commercial compounds}

The triple interaction between storage temperature, storage period and foliar application had a significant effect on germination percentage in both seasons. The highest germination percentage was recorded for the treatment with boron or amica in all storage periods at $5^{\circ} \mathrm{C}$ compared with all other different treatments in the first and second seasons, respectively as shown in Table $\mathbf{1 0}$.

\section{Water content}

\section{Effect of storage temperature on water content}

Storage temperature had a significant effect on water content of seeds in both seasons of the study. Storage at $5^{\circ} \mathrm{C}$ resulted in higher water content than storage at room temperature as shown Table 7. These results are in agreement with those obtained by Stanwood and Sowa (1995) and Yanping et al. (1999).

\section{Effect of storage period on water content}

It is clear from data presented in Table $\mathbf{7}$ that the water content was significantly increased with prolongation of the storage periods. The water content started to increase slowly and successively increased till the end of storage. These results are in agreement with those obtained by Kavak and Eser (2009) who found that water content of onion seed had raised from $9 \%$ to $13 \%$ during storage at $25^{\circ} \mathrm{C}$ for 10 weeks.

\section{Effect of foliar spraying with commercial compounds on water content}

Concerning the effect of foliar application with commercial compounds on water content of seeds, data in Table 7 show that the lowest values of water content was 
Table 10: Effect of the interaction between storage temperature, storage period and foliar application with commercial compounds on germination (\%) of onion seeds during seasons of 2008/2009 and 2009/2010

\begin{tabular}{|c|c|c|c|c|c|c|c|c|}
\hline \multirow[t]{3}{*}{ Interactions } & \multicolumn{8}{|c|}{ Storage period (month) } \\
\hline & $\mathbf{0}$ & 4 & 8 & 12 & $\mathbf{0}$ & 4 & 8 & 12 \\
\hline & \multicolumn{4}{|c|}{ Season $2008 / 2009$} & \multicolumn{4}{|c|}{ Season $2009 / 2010$} \\
\hline & \multicolumn{8}{|c|}{ Room temperature } \\
\hline Union Zn & 93.75 & 88.0 & 77.5 & 72.5 & 93.00 & 85.75 & 79.00 & 76.75 \\
\hline Union Feer & 96.50 & 87.2 & 80.5 & 75.5 & 95.50 & 89.00 & 83.25 & 70.75 \\
\hline Union Mn & 95.25 & 85.5 & 76 & 71.7 & 94.75 & 87.75 & 80.00 & 80.25 \\
\hline Shams K & 91.50 & 82.5 & 78.5 & 64.5 & 95.50 & 90.75 & 80.25 & 69.75 \\
\hline Boron & 99.50 & 84.2 & 76.7 & 71.7 & 96.75 & 89.25 & 80.00 & 75.50 \\
\hline Magnesium & 91.25 & 79.0 & 66.2 & 61.0 & 92.50 & 86.25 & 73.75 & 65.75 \\
\hline Elga 600 & 94.50 & 83.7 & 72.2 & 67.7 & 94.25 & 85.75 & 78.50 & 64.50 \\
\hline Caboron & 96.00 & 85.5 & 79.5 & 74.5 & 94.25 & 91.50 & 77.75 & 75.00 \\
\hline Amica & 93.75 & 88.7 & 80 & 75 & 93.50 & 90.00 & 80.25 & 71.75 \\
\hline Shetocare & 95.50 & 81.2 & 70.5 & 65.5 & 94.25 & 89.75 & 78.50 & 66.50 \\
\hline Hummer & 92.50 & 89.2 & 82 & 77 & 93.00 & 87.00 & 82.25 & 73.75 \\
\hline Amino $\mathrm{X}$ & 96.25 & 87 & 78.2 & 73.2 & 93.25 & 89.75 & 82.75 & 75.75 \\
\hline Untreated & 92.00 & 80 & 71.2 & 68.7 & 92.75 & 87.00 & 75.25 & 65.75 \\
\hline \multicolumn{9}{|c|}{ Cold temperature $\left(5^{\circ} \mathrm{C}\right)$} \\
\hline Union Zn & 93.75 & 90.2 & 83.5 & 78.5 & 93.00 & 92.00 & 83.50 & 78.50 \\
\hline Union Feer & 96.50 & 88.2 & 81 & 76.0 & 95.50 & 93.50 & 86.75 & 81.75 \\
\hline Union Mn & 95.25 & 89.7 & 84.2 & 79.2 & 94.75 & 92.50 & 82.50 & 77.50 \\
\hline Shams K & 91.50 & 89.5 & 85 & 80.0 & 95.50 & 91.50 & 78.75 & 72.25 \\
\hline Boron & 99.50 & 93 & 90.3 & 84.2 & 96.75 & 93.00 & 83.25 & 79.00 \\
\hline Magnesium & 91.25 & 88 & 80.7 & 75.7 & 92.50 & 89.25 & 79.25 & 73.00 \\
\hline Elga 600 & 94.50 & 88.5 & 82.7 & 77.7 & 94.25 & 92.50 & 87.00 & 82.00 \\
\hline Caboron & 96.00 & 86.2 & 80.2 & 75.2 & 94.25 & 93.00 & 85.25 & 80.25 \\
\hline Amica & 93.75 & 81.5 & 88.5 & 83.5 & 93.50 & 90.25 & 87.00 & 82.50 \\
\hline Shetocare & 95.50 & 85.0 & 77 & 72.0 & 94.25 & 91.00 & 80.25 & 74.25 \\
\hline Hummer & 92.50 & 85.2 & 76.2 & 71.5 & 93.00 & 92.50 & 83.50 & 78.50 \\
\hline Amino $\mathrm{X}$ & 96.25 & 88.2 & 83.5 & 76 & 93.25 & 91.75 & 84.50 & 79.50 \\
\hline Untreated & 92.00 & 86.5 & 79.5 & 74.5 & 92.75 & 88.75 & 83.25 & 76.75 \\
\hline
\end{tabular}

Details of treatment listed in Table 3

Table 11: Effect of the interaction between storage temperature and storage period on water content (\%) of onion seeds during seasons of $2008 / 2009$ and 2009/2010

\begin{tabular}{|c|c|c|c|c|}
\hline \multirow{3}{*}{$\begin{array}{l}\text { Storage period } \\
\text { (month) }\end{array}$} & \multicolumn{4}{|c|}{ Storage temperature } \\
\hline & Room temperature & Cold temp. $\left(5^{\circ} \mathrm{C}\right)$ & Room temperature & Cold temp. $\left(5^{\circ} \mathrm{C}\right)$ \\
\hline & \multicolumn{2}{|c|}{ Season $2008 / 2009$} & \multicolumn{2}{|c|}{ Season $2009 / 2010$} \\
\hline $\mathbf{0}$ & $4.17 \mathrm{e}$ & $4.17 \mathrm{e}$ & $4.20 \mathrm{f}$ & $4.20 \mathrm{f}$ \\
\hline 4 & $4.52 \mathrm{c}$ & $4.42 \mathrm{~d}$ & $5.08 \mathrm{a}$ & $4.83 \mathrm{~b}$ \\
\hline 8 & $4.17 \mathrm{e}$ & $6.36 \mathrm{a}$ & $4.66 \mathrm{c}$ & $4.67 \mathrm{c}$ \\
\hline 12 & $3.69 \mathrm{f}$ & $5.79 \mathrm{~b}$ & $4.39 \mathrm{e}$ & $4.58 \mathrm{~d}$ \\
\hline LSD $_{0.05}$ & 0.0975 & & 0.0624 & \\
\hline
\end{tabular}

recorded for treatments with union feer, shams $\mathrm{K}$ or boron in the first season, and union feer, shams $\mathrm{K}$, boron, magnesium, shetocare or hummer in the second season.

\section{Effect of the interaction between storage temperature and storage period}

The effect of the interaction between storage temperature and storage period on water content, data in Table 11 shows that water content $(\%)$ increased and reached to the maximum value after 4 months of storage at room temperature and after 8 months of storage at $5^{\circ} \mathrm{C}$ in the first season. Meanwhile, water content increased and reached to the maximum value after 4 months of storage at both storage temperatures in the second season.
Effect of the interaction between storage temperature and foliar application with commercial compounds

It is clear from data presented in Table $\mathbf{1 2}$ that water content at $5^{\circ} \mathrm{C}$ is higher than that at room temperature in all tested treatments. The highest water content was recorded for the control at $5^{\circ} \mathrm{C}$ in the first season and union $\mathrm{Zn}$, amica or the control at $5^{\circ} \mathrm{C}$ in the second season.

\section{Effect of the interaction between storage periods and foliar application with commercial compound}

With respect to the effect of the interaction between storage periods and foliar application on water content, data in Table 13 show that water content gradually increased in all tested treatments as storage period increased. The 
Table 12: Effect of the interaction between storage temperature and foliar application with commercial compound on water content (\%) of onion seeds during seasons of 2008/2009 and 2009/2010

\begin{tabular}{|c|c|c|c|c|}
\hline \multirow[t]{3}{*}{ Treatments } & \multicolumn{4}{|c|}{ Storage temperature } \\
\hline & Room temperature & Cold temp. $\left(5^{\circ} \mathrm{C}\right)$ & Room temperature & Cold temp. $\left(5^{\circ} \mathrm{C}\right)$ \\
\hline & \multicolumn{2}{|c|}{ Season $2008 / 2009$} & \multicolumn{2}{|c|}{ Season $2009 / 2010$} \\
\hline Union $\mathrm{Zn}$ & $4.13 \mathrm{f}-\mathrm{g}$ & $5.11 \mathrm{c}-\mathrm{e}$ & $4.60 \mathrm{a}-\mathrm{g}$ & $4.71 \mathrm{a}$ \\
\hline Union Feer & $4.02 \mathrm{~g}$ & $5.15 \mathrm{~b}-\mathrm{e}$ & $4.57 \mathrm{~b}-\mathrm{h}$ & $4.57 \mathrm{~b}-\mathrm{h}$ \\
\hline Union Mn & $4.13 \mathrm{f}-\mathrm{g}$ & $5.23 \mathrm{a}-\mathrm{c}$ & 4.61 a-f & $4.60 \mathrm{a}-\mathrm{g}$ \\
\hline Shams K & $4.21 \mathrm{f}$ & $5.00 \mathrm{e}$ & $4.46 \mathrm{~h}-\mathrm{j}$ & $4.55 \mathrm{c}-\mathrm{i}$ \\
\hline Boron & $4.08 \mathrm{f}-\mathrm{g}$ & $5.05 \mathrm{~d}-\mathrm{e}$ & $4.53 \mathrm{e}-\mathrm{i}$ & $4.49 \mathrm{f}-\mathrm{j}$ \\
\hline Magnesium & $4.10 \mathrm{f}-\mathrm{g}$ & $5.23 \mathrm{a}-\mathrm{c}$ & $4.48 \mathrm{~g}-\mathrm{j}$ & $4.40 \mathrm{j}$ \\
\hline Elga 600 & $4.10 \mathrm{f}-\mathrm{g}$ & $5.24 \mathrm{a}-\mathrm{c}$ & $4.67 \mathrm{a}-\mathrm{d}$ & $4.53 \mathrm{e}-\mathrm{i}$ \\
\hline Caboron & $4.18 \mathrm{f}-\mathrm{g}$ & $5.10 \mathrm{c}-\mathrm{e}$ & 4.66 a-e & 4.60 a-e \\
\hline Amica & $4.21 \mathrm{f}$ & $5.27 \mathrm{a}-\mathrm{b}$ & $4.67 \mathrm{a}-\mathrm{d}$ & $4.70 \mathrm{a}-\mathrm{b}$ \\
\hline Shetocare & $4.20 \mathrm{f}$ & $5.23 \mathrm{a}-\mathrm{c}$ & $4.43 \mathrm{i}-\mathrm{j}$ & $4.48 \mathrm{~g}-\mathrm{i}$ \\
\hline Hummer & $4.16 \mathrm{f}-\mathrm{g}$ & $5.28 \mathrm{a}-\mathrm{b}$ & $4.50 \mathrm{f}-\mathrm{j}$ & $4.58 \mathrm{a}-\mathrm{g}$ \\
\hline Amino $\mathrm{X}$ & $4.16 \mathrm{f}-\mathrm{g}$ & $5.21 \mathrm{a}-\mathrm{d}$ & $4.70 a-b$ & $4.54 \mathrm{~d}-\mathrm{i}$ \\
\hline Untreated & $4.15 \mathrm{f}-\mathrm{g}$ & $5.32 \mathrm{a}$ & $4.68 \mathrm{a}-\mathrm{c}$ & $4.60 \mathrm{a}-\mathrm{d}$ \\
\hline LSD $_{0.05}$ & \multicolumn{2}{|c|}{0.161} & \multicolumn{2}{|c|}{0.132} \\
\hline
\end{tabular}

Details of treatment listed in Table 3

Table 13: Effect of the interaction between storage period and foliar application with commercial compounds on water content (\%) of onion seeds during 2008/2009 season

\begin{tabular}{|c|c|c|c|c|c|c|c|c|}
\hline \multirow[t]{3}{*}{ Treatment } & \multicolumn{8}{|c|}{ Storage period (month) } \\
\hline & $\mathbf{0}$ & 4 & 8 & 12 & $\mathbf{0}$ & 4 & 8 & 12 \\
\hline & \multicolumn{4}{|c|}{ Season $2008 / 2009$} & \multicolumn{4}{|c|}{ Season $2009 / 2010$} \\
\hline Union $\mathrm{Zn}$ & $4.25 \mathrm{j}-\mathrm{n}$ & $4.42 \mathrm{~g}-\mathrm{k}$ & $5.17 \mathrm{a}$ & $4.65 \mathrm{~b}-\mathrm{g}$ & 4.27 p-r & $5.06 \mathrm{a}$ & $4.75 \mathrm{e}-\mathrm{h}$ & $4.55 \mathrm{i}-\mathrm{n}$ \\
\hline Union Feer & $3.92 \mathrm{o}$ & $4.32 \mathrm{i}-\mathrm{m}$ & $5.23 \mathrm{a}$ & $4.87 \mathrm{~b}$ & $4.07 \mathrm{t}-\mathrm{V}$ & $4.97 \mathrm{a}-\mathrm{c}$ & $4.72 \mathrm{f}-\mathrm{i}$ & $4.52 \mathrm{j}-\mathrm{n}$ \\
\hline Union Mn & $4.15 \mathrm{~m}-\mathrm{o}$ & $4.38 \mathrm{~h}-1$ & $5.38 \mathrm{a}$ & $4.80 \mathrm{~b}-\mathrm{c}$ & $4.22 \mathrm{q}-\mathrm{u}$ & $4.98 \mathrm{a}-\mathrm{b}$ & $4.72 \mathrm{f}-\mathrm{i}$ & $4.52 \mathrm{k}-\mathrm{O}$ \\
\hline Shams K & $4.25 \mathrm{j}-\mathrm{n}$ & $4.47 \mathrm{f}-\mathrm{j}$ & $5.17 \mathrm{a}$ & $4.53 \mathrm{~d}-\mathrm{i}$ & $4.05 \mathrm{u}-\mathrm{v}$ & 4.91a-e & $4.68 \mathrm{f}-\mathrm{k}$ & $4.38 \mathrm{n}-\mathrm{q}$ \\
\hline Boron & $4.02 \mathrm{n}-\mathrm{o}$ & $4.40 \mathrm{~h}-1$ & $5.20 \mathrm{a}$ & $4.66 \mathrm{~b}-\mathrm{f}$ & $4.17 \mathrm{r}-\mathrm{V}$ & $4.83 b-f$ & $4.61 \mathrm{~g}-1$ & $4.431-\mathrm{p}$ \\
\hline Magnesium & $4.12 \mathrm{~m}-\mathrm{o}$ & $4.46 \mathrm{f}-\mathrm{j}$ & $5.22 \mathrm{a}$ & $4.86 \mathrm{~b}$ & $4.12 \mathrm{~s}-\mathrm{V}$ & $4.78 \mathrm{~d}-\mathrm{g}$ & $4.51 \mathrm{k}-\mathrm{o}$ & $4.33 \mathrm{o}-\mathrm{r}$ \\
\hline Elga 600 & $4.12 \mathrm{~m}-\mathrm{o}$ & $4.40 \mathrm{~h}-1$ & $5.40 \mathrm{a}$ & $4.76 \mathrm{~b}-\mathrm{d}$ & $4.20 \mathrm{r}-\mathrm{v}$ & $4.98 \mathrm{a}-\mathrm{b}$ & $4.68 \mathrm{f}-\mathrm{k}$ & $4.55 \mathrm{i}-\mathrm{n}$ \\
\hline Caboron & $4.171-n$ & $4.52 \mathrm{e}-\mathrm{i}$ & $5.21 \mathrm{a}$ & $4.65 \mathrm{~b}-\mathrm{g}$ & $4.40 \mathrm{~m}-\mathrm{q}$ & $4.96 \mathrm{a}-\mathrm{d}$ & $4.68 \mathrm{f}-\mathrm{k}$ & $4.58 \mathrm{~h}-1$ \\
\hline Amica & $4.27 \mathrm{j}-\mathrm{m}$ & $4.51 \mathrm{e}-\mathrm{i}$ & $5.33 \mathrm{a}$ & $4.86 \mathrm{~b}$ & $4.451 \mathrm{~m}-\mathrm{p}$ & $5.08 \mathrm{a}$ & $4.71 \mathrm{f}-\mathrm{i}$ & $4.501-\mathrm{O}$ \\
\hline Shetocare & $4.22 \mathrm{k}-\mathrm{n}$ & $4.57 \mathrm{c}-\mathrm{h}$ & $5.32 \mathrm{a}$ & $4.76 \mathrm{~b}-\mathrm{d}$ & $4.02 \mathrm{v}$ & $4.80 \mathrm{c}-\mathrm{f}$ & $4.56 \mathrm{i}-\mathrm{n}$ & $4.431-\mathrm{O}$ \\
\hline Hummer & $4.32 \mathrm{i}-\mathrm{m}$ & $4.53 \mathrm{~d}-\mathrm{i}$ & $5.31 \mathrm{a}$ & $4.72 \mathrm{~b}-\mathrm{e}$ & $4.12 \mathrm{~s}-\mathrm{v}$ & $5.00 \mathrm{a}-\mathrm{b}$ & $4.57 \mathrm{~h}-\mathrm{m}$ & $4.461-0$ \\
\hline Amino $\mathrm{X}$ & $4.25 \mathrm{j}-\mathrm{n}$ & $4.46 \mathrm{f}-\mathrm{j}$ & $5.25 \mathrm{a}$ & $4.80 \mathrm{~b}-\mathrm{c}$ & $4.22 \mathrm{q}-\mathrm{u}$ & $5.03 \mathrm{a}$ & $4.70 \mathrm{f}-\mathrm{j}$ & $4.52 \mathrm{j}-\mathrm{o}$ \\
\hline Untreated & $4.22 \mathrm{k}-\mathrm{n}$ & 4.66 b-f & $5.39 \mathrm{a}$ & $4.76 \mathrm{~b}-\mathrm{d}$ & $4.25 \mathrm{q}-\mathrm{t}$ & $5.03 \mathrm{a}$ & $4.72 \mathrm{f}-\mathrm{i}$ & $4.56 \mathrm{i}-\mathrm{o}$ \\
\hline LSD $_{0.05}$ & \multicolumn{4}{|c|}{0.228} & \multicolumn{4}{|c|}{0.186} \\
\hline
\end{tabular}

Details of treatment listed in Table 3

highest water content was recorded for all tested treatments after 8 months of storage and the treatment with union feer, magnesium or amica after 12 months of storage in the first season. The highest water content after 12 months of storage was recorded for the treatment with union $\mathrm{Zn}$, elga 600 or the control in the second season.

\section{Effect of the interaction between storage temperature, storage period and foliar application with commercial compounds}

The triple interaction between storage temperature, storage period and foliar application had a significant effect on water content in both seasons. The highest water content of onion seeds was recorded for treatments with union Mn, elga 600, amica, shetocare, hummer, magnesium or the untreated in the first season after 8 months of storage at $5^{\circ} \mathrm{C}$. Meanwhile, the highest water content in the second season was recorded for treatments with elga 600, amica, amino X or the untreated after 4 months of storage at room temperature as shown in Table 14.

\section{Storage of onion seed and antioxidants}

Consuming vegetables and fruits may reduce the risk of chronic diseases, including cardiovascular disease, stroke, neurodegeneration, and type II diabetes. Substantial recent research has been performed to investigate the potential health benefits of antioxidants in food. Antioxidants can inhibit oxidative reactions in vivo, and aid in functional performance of enzyme systems for self-defence mechanisms within cells. Among all vegetables, onion is a species consumed widely across the world and possesses a high content of flavonoid compounds. Onions contain high levels of flavonoids, a major class of non-nutrient antioxidants (quercetin glycosides), anthocyanins (cyaniding glycosides) and sulphur compounds (i.e. thiosulphinates), both of which have a high level of antioxidant activity These compounds are effective scavengers of free radicals that are thought to induce DNA damage and tumor promotion. Flavonoids, in general, also have preventive effects on a 
Table 14: Effect of the interaction between storage temperature, storage period and foliar application with commercial compounds on water content ( $\%$ ) of onion seeds during seasons of 2008/2009 and 2009/2010

\begin{tabular}{|c|c|c|c|c|c|c|c|c|}
\hline \multirow{3}{*}{ Interactions } & \multicolumn{8}{|c|}{ Storage period (month) } \\
\hline & $\mathbf{0}$ & 4 & 8 & 12 & $\mathbf{0}$ & 4 & 8 & 12 \\
\hline & \multicolumn{4}{|c|}{ Season $2008 / 2009$} & \multicolumn{4}{|c|}{ Season $2009 / 2010$} \\
\hline & \multicolumn{8}{|c|}{ Room temperature } \\
\hline Union Zn & 4.25 & 4.50 & 4.17 & 3.62 & 4.275 & 5.175 & 4.625 & 4.350 \\
\hline Union Feer & 3.92 & 4.32 & 4.17 & 3.67 & 4.075 & 5.100 & 4.700 & 4.425 \\
\hline Union Mn & 4.15 & 4.52 & 4.25 & 3.60 & 4.225 & 5.075 & 4.750 & 4.425 \\
\hline Shams K & 4.25 & 4.60 & 4.25 & 3.75 & 4.050 & 4.950 & 4.575 & 4.300 \\
\hline Boron & 4.02 & 4.42 & 4.17 & 3.72 & 4.175 & 4.950 & 4.675 & 4.350 \\
\hline Magnesium & 4.12 & 4.50 & 4.02 & 3.75 & 4.125 & 4.925 & 4.525 & 4.350 \\
\hline Elga 600 & 4.12 & 4.42 & 4.17 & 3.67 & 4.200 & 5.200 & 4.750 & 4.550 \\
\hline Caboron & 4.17 & 4.55 & 4.20 & 3.80 & 4.400 & 5.075 & 4.725 & 4.475 \\
\hline Amica & 4.27 & 4.70 & 4.15 & 3.75 & 4.450 & 5.200 & 4.725 & 4.325 \\
\hline Shetocare & 4.22 & 4.67 & 4.25 & 3.67 & 4.025 & 4.925 & 4.450 & 4.325 \\
\hline Hummer & 4.32 & 4.55 & 4.12 & 3.65 & 4.125 & 5.125 & 4.500 & 4.250 \\
\hline Amino $\mathrm{X}$ & 4.25 & 4.50 & 4.17 & 3.75 & 4.225 & 5.200 & 4.825 & 4.550 \\
\hline Untreated & 4.22 & 4.55 & 4.20 & 3.62 & 4.250 & 5.200 & 4.775 & 4.500 \\
\hline \multicolumn{9}{|c|}{ Cold temperature $\left(5^{\circ} \mathrm{C}\right)$} \\
\hline Union Zn & 4.25 & 4.35 & 6.17 & 5.67 & 4.275 & 4.950 & 4.875 & 4.750 \\
\hline Union Feer & 3.92 & 4.32 & 6.30 & 6.07 & 4.075 & 4.850 & 4.753 & 4.625 \\
\hline Union Mn & 4.15 & 4.25 & 6.52 & 6.00 & 4.225 & 4.900 & 4.700 & 4.600 \\
\hline Shams K & 4.25 & 4.35 & 6.10 & 5.32 & 4.050 & 4.875 & 4.800 & 4.475 \\
\hline Boron & 4.02 & 4.37 & 6.22 & 5.60 & 4.175 & 4.725 & 4.550 & 4.525 \\
\hline Magnesium & 4.12 & 4.42 & 6.42 & 5.97 & 4.125 & 4.650 & 4.500 & 4.325 \\
\hline Elga 600 & 4.12 & 4.37 & 6.62 & 5.85 & 4.200 & 4.775 & 4.625 & 4.550 \\
\hline Caboron & 4.17 & 4.50 & 6.22 & 5.50 & 4.400 & 4.850 & 4.650 & 4.700 \\
\hline Amica & 4.27 & 4.32 & 6.52 & 5.97 & 4.450 & 4.975 & 4.700 & 4.675 \\
\hline Shetocare & 4.22 & 4.47 & 6.40 & 5.85 & 4.025 & 4.675 & 4.675 & 4.550 \\
\hline Hummer & 4.32 & 4.52 & 6.50 & 5.80 & 4.125 & 4.875 & 4.650 & 4.675 \\
\hline Amino $X$ & 4.25 & 4.42 & 6.32 & 5.85 & 4.225 & 4.875 & 4.575 & 4.500 \\
\hline Untreated & 4.22 & 4.77 & 6.40 & 5.90 & 4.250 & 4.875 & 4.675 & 4.625 \\
\hline
\end{tabular}

Details of treatment listed in Table 3

number of degenerative pathologies such as cardiovascular and neurological diseases, and other dysfunctions related to oxidative stresses ( $\mathrm{Lu}$ et al. 2011).

There has been increasing interest for the inclusion of fresh fruits and vegetables in the human diet, mainly for the health benefits associated with their consumption. A major benefit from a higher intake of fruits and vegetables may be the increased consumption of vitamins (vitamin C, vitamin $\mathrm{A}$, vitamin $\mathrm{B} 6$, thiamin, and niacin), minerals, and dietary fiber. Other constituents that may lower the risk of cancer and heart disease as well as prevent degenerative diseases include antioxidant compounds such as carotenoids, flavonoids, and other phenolics. These compounds are found ubiquitously in edible plants and are important constituents of the human diet. Epidemiologic studies that analyze the health implications of dietary components must estimate the intake in sample populations. Therefore, the availability of appropriate and complete food composition data is crucial. Due to the chemical diversity of antioxidant compounds present in foods, complete databases on food antioxidant content are not yet available (Kevers et al. 2007).

Several studies have addressed the changes in the phenolic compounds during storage of different fruits and vegetables. Generally, these results show that antioxidant activity and concentration of phenolics often increases during storage (Zhang et al. 2008; Kevers et al. 2007), although a few studies report constant or decreasing levels during storage (Rodrigues et al. 2010).

\section{Catalase activity}

\section{Effect of storage temperature on catalase activity}

It is clear from data presented in Table 15 that there was non significant difference of storage temperature on catalase activity in both seasons but there was a slight increase in catalase activity in seed stored at $5^{\circ} \mathrm{C}$ than in room temperature. These results are in agreement with those of Yanping et al. (2000) and Demirkaya et al. (2010). They found that the antioxidant enzymes activity was higher in cold storage than in room temperature.

\section{Effect of storage period on catalase activity}

Storage period had a significant effect on catalase activity in both seasons. It is clear from data presented in Table $\mathbf{1 5}$ that catalase activity was significantly decreased as storage period increased. These results are in agreement with Golovina et al. (1997) and Demirkaya et al. (2010).

\section{Effect of foliar application with commercial compounds on catalase activity}

Concerning the effect of foliar application with commercial compounds on catalase activity, data in Table $\mathbf{1 5}$ show that the treatment with shams $\mathrm{K}$, boron, shetocare 
Table 15: Effect of storage temperature, storage period and foliar application with commercial compounds on catalase and peroxidase activity of onion seeds during seasons of $2008 / 2009$ and 2009/2010

\begin{tabular}{|c|c|c|c|c|}
\hline \multirow[t]{2}{*}{ Treatments } & \multicolumn{2}{|c|}{ Catalase activity (enzyme unit) } & \multicolumn{2}{|c|}{ Peroxidase Activity (enzyme unit) } \\
\hline & $2008 / 2009$ & $2009 / 2010$ & $2008 / 2009$ & $2009 / 2010$ \\
\hline $\begin{array}{l}\text { Storage temperature } \\
\text { Room temperature } \\
\text { Cold temperature }\left(5^{\circ} \mathrm{C}\right) \\
\text { LSD }_{\mathbf{0 . 0 5}}\end{array}$ & $\begin{array}{c}12.98 \\
13.71 \\
\text { N.S. }\end{array}$ & $\begin{array}{c}13.08 \\
14.24 \\
\text { N.S. }\end{array}$ & $\begin{array}{c}8.573 \\
8.833 \\
\text { N.S. }\end{array}$ & $\begin{array}{c}8.578 \\
9.117 \\
\text { N.S. }\end{array}$ \\
\hline $\begin{array}{c}\text { Storage periods (month) } \\
0 \\
6 \\
12 \\
\text { LSD }_{0.05} \\
\end{array}$ & $\begin{array}{l}17.79 \mathrm{a} \\
13.40 \mathrm{~b} \\
8.832 \mathrm{c} \\
\mathbf{0 . 9 0 8}\end{array}$ & $\begin{array}{l}16.54 \mathrm{a} \\
12.80 \mathrm{~b} \\
11.65 \mathrm{c} \\
\mathbf{0 . 8 8 7 1}\end{array}$ & $\begin{array}{l}11.19 \mathrm{a} \\
8.863 \mathrm{~b} \\
6.059 \mathrm{c} \\
\mathbf{0 . 6 1 9 5}\end{array}$ & $\begin{array}{l}10.66 \mathrm{a} \\
8.600 \mathrm{~b} \\
7.281 \mathrm{c} \\
\mathbf{0 . 9 1 5 9}\end{array}$ \\
\hline $\begin{array}{l}\text { Foliar application } \\
\text { Union } \mathrm{Zn} \\
\text { Union Feer } \\
\text { Union } \mathrm{Mn} \\
\text { Shams } \mathrm{K} \\
\text { Boron } \\
\text { Magnesium } \\
\text { Elga } 600 \\
\text { Caboron } \\
\text { Amica } \\
\text { Shetocare } \\
\text { Hummer } \\
\text { Amino X } \\
\text { Untreated } \\
\text { LSD }\end{array}$ & $\begin{array}{c}11.53 \mathrm{~d}-f \\
13.81 \mathrm{~b}-\mathrm{d} \\
12.09 \mathrm{c}-\mathrm{f} \\
14.55 \mathrm{a}-\mathrm{b} \\
16.96 \mathrm{a} \\
10.84 \mathrm{e}-\mathrm{f} \\
10.25 \mathrm{f} \\
14.05 \mathrm{~b}-\mathrm{c} \\
14.12 \mathrm{~b}-\mathrm{c} \\
14.67 \mathrm{a}-\mathrm{b} \\
12.85 \mathrm{~b}-\mathrm{e} \\
14.60 \mathrm{a}-\mathrm{b} \\
11.12 \mathrm{~d}-\mathrm{f} \\
\mathbf{2 . 4 6 2}\end{array}$ & $\begin{array}{c}14.23 \mathrm{~b}-\mathrm{e} \\
13.13 \mathrm{~d} \\
12.97 \mathrm{~d} \\
15.85 \mathrm{a}-\mathrm{b} \\
16.82 \mathrm{a} \\
10.20 \mathrm{e} \\
12.94 \mathrm{~d} \\
12.75 \mathrm{~d} \\
12.06 \mathrm{~d}-\mathrm{e} \\
15.67 \mathrm{a}-\mathrm{c} \\
12.06 \mathrm{~d}-\mathrm{e} \\
15.47 \mathrm{a}-\mathrm{c} \\
13.45 \mathrm{c}-\mathrm{d} \\
\mathbf{2 . 2 1 9}\end{array}$ & $\begin{array}{l}7.487 \text { d-e } \\
10.43 \text { a-b } \\
9.733 \text { a-c } \\
8.359 \text { c-d } \\
10.69 \text { a } \\
5.853 \mathrm{e} \\
9.363 \mathrm{a}-\mathrm{c} \\
8.289 \mathrm{c}-\mathrm{d} \\
8.404 \mathrm{c}-\mathrm{d} \\
9.491 \mathrm{a}-\mathrm{c} \\
7.423 \mathrm{~d}-\mathrm{e} \\
8.754 \mathrm{~b}-\mathrm{d} \\
8.858 \mathrm{~b}-\mathrm{d} \\
\mathbf{1 . 7 4 9}\end{array}$ & $\begin{array}{c}7.576 \mathrm{~d}-\mathrm{f} \\
9.434 \mathrm{a}-\mathrm{c} \\
11.02 \mathrm{a} \\
8.918 \mathrm{~b}-\mathrm{d} \\
10.61 \mathrm{a} \\
6.704 \mathrm{ef} \\
9.616 \mathrm{a}-\mathrm{c} \\
6.120 \mathrm{f} \\
8.861 \mathrm{c}-\mathrm{d} \\
10.55 \mathrm{a}-\mathrm{b} \\
7.972 \mathrm{c}-\mathrm{e} \\
9.616 \mathrm{a}-\mathrm{c} \\
8.117 \mathrm{c}-\mathrm{e} \\
\mathbf{1 . 6 5 0}\end{array}$ \\
\hline
\end{tabular}

Details of treatment listed in Table 3

Table 16: Effect of the interaction between storage temperature and storage period on catalase activity of onion seeds during seasons of $2008 / 2009$ and $2009 / 2010$

\begin{tabular}{|c|c|c|c|c|}
\hline \multirow{3}{*}{$\begin{array}{l}\text { Storage period } \\
\text { (month) }\end{array}$} & \multicolumn{4}{|c|}{ Storage temperature } \\
\hline & Room temperature & Cold temp. $\left(5^{\circ} \mathrm{C}\right)$ & Room temperature & Cold temp. $\left(5^{\circ} \mathrm{C}\right)$ \\
\hline & \multicolumn{2}{|c|}{ Season $2008 / 2009$} & \multicolumn{2}{|c|}{ Season $2009 / 2010$} \\
\hline $\begin{array}{c}0 \\
6 \\
12 \\
\text { LSD }\end{array}$ & $\begin{array}{l}17.79 \mathrm{a} \\
13.42 \mathrm{~b} \\
7.724 \mathrm{~d} \\
\mathbf{1 . 2 8 5}\end{array}$ & $\begin{array}{l}17.79 \mathrm{a} \\
13.38 \mathrm{~b} \\
9.941 \mathrm{c}\end{array}$ & $\begin{array}{c}16.54 \mathrm{a} \\
12.43 \mathrm{~b} \\
10.27 \mathrm{c} \\
\mathbf{1 . 2 5 4}\end{array}$ & $\begin{array}{l}16.54 \mathrm{a} \\
13.17 \mathrm{~b} \\
13.02 \mathrm{~b}\end{array}$ \\
\hline
\end{tabular}

or amino $\mathrm{X}$ had the highest catalase activity in both seasons. These treatments had the highest catalase activity after harvest and they had an important role on enzyme activation. These results are in agreement with those of Ilbi and Eser (2002), Pablo and William (2005) and Heidari and Jamashidi (2011).

\section{Effect of the interaction between storage temperature and storage period}

The results illustrated in Table 16 indicate that catalase activity in all storage periods decreased as storage temperature increased in both seasons. The lowest value of catalase was recorded after 12 months of storage at room temperature in both seasons.

\section{Effect of the interaction between storage temperature and foliar application with commercial compounds}

Concerning the effect of the interaction between storage temperatures and foliar application on catalase activity, the highest value of catalase was recorded for boron treatment at both storage temperatures in the first season, and the treatment with boron or shetocare at $5^{\circ} \mathrm{C}$ in the second season as shown in Table 17.

\section{Effect of the interaction between storage period and foliar application with commercial compounds}

Data in Table 18 show that the interaction between storage period and foliar application had significant effect on catalase activity. Catalase activity in different treatments of foliar spray was gradually decreased as storage period increased. The highest catalase activity after 12 months of storage was recorded for the treatment with boron or amica in the first season, and the treatment with boron in the second season.

\section{Effect of the interaction between storage temperature, storage period and foliar spraying with commercial products}

The effects of interaction between storage temperature, storage period and foliar application with commercial compounds on catalase activity are shown in Table 19 show 
Table 17a: Effect of the interaction between storage temperature and foliar application with commercial compounds on catalase and peroxidase activity of onion seeds during growing seasons

\begin{tabular}{|c|c|c|c|c|c|c|}
\hline \multirow[t]{3}{*}{ Treatment } & \multicolumn{4}{|c|}{ Catalase activity (enzyme unit) } & \multicolumn{2}{|c|}{ Peroxidase activity } \\
\hline & Room temp. & $5^{\circ} \mathbf{C}$ & Room temp. & $5^{\circ} \mathbf{C}$ & Room temp. & $5^{\circ} \mathbf{C}$ \\
\hline & \multicolumn{2}{|c|}{ Season 2008 / 2009} & \multicolumn{2}{|c|}{ Season 2009 / 2010} & \multicolumn{2}{|c|}{ Season 2008 / 2009} \\
\hline Union Zn & $11.65 \mathrm{f}-\mathrm{h}$ & $11.41 \mathrm{f}-\mathrm{h}$ & 14.82 b-f & $13.65 \mathrm{c}-\mathrm{g}$ & $7.02 \mathrm{f}-\mathrm{h}$ & $7.94 \mathrm{c}-\mathrm{h}$ \\
\hline Union Feer & $13.88 \mathrm{a}-\mathrm{g}$ & $13.73 \mathrm{a}-\mathrm{g}$ & $13.15 \mathrm{c}-\mathrm{h}$ & $13.12 \mathrm{c}-\mathrm{h}$ & $10.35 a-c$ & $10.51 \mathrm{a}-\mathrm{b}$ \\
\hline Union Mn & $11.86 \mathrm{e}-\mathrm{h}$ & $12.31 \mathrm{~d}-\mathrm{h}$ & $12.61 \mathrm{e}-\mathrm{i}$ & $13.33 \mathrm{c}-\mathrm{h}$ & $9.92 \mathrm{a}-\mathrm{c}$ & 9.54 a-e \\
\hline Shams K & $13.72 \mathrm{a}-\mathrm{g}$ & $15.38 \mathrm{a}-\mathrm{d}$ & $15.81 \mathrm{~b}-\mathrm{d}$ & $15.89 \mathrm{~b}-\mathrm{c}$ & $7.90 \mathrm{c}-\mathrm{h}$ & 8.81 a-f \\
\hline Boron & $16.73 a-b$ & $17.19 \mathrm{a}$ & $13.91 \mathrm{c}-\mathrm{g}$ & $19.74 \mathrm{a}$ & $10.26 \mathrm{a}-\mathrm{c}$ & $11.12 \mathrm{a}$ \\
\hline Magnesium & $10.59 \mathrm{~g}-\mathrm{h}$ & $11.10 \mathrm{f}-\mathrm{h}$ & $9.91 \mathrm{i}$ & $10.48 \mathrm{~h}-\mathrm{i}$ & $5.84 \mathrm{~h}$ & $5.86 \mathrm{~g}-\mathrm{h}$ \\
\hline Elga 600 & $9.461 \mathrm{~h}$ & $11.04 \mathrm{f}-\mathrm{h}$ & $12.35 \mathrm{e}-\mathrm{i}$ & $13.54 \mathrm{c}-\mathrm{h}$ & $9.47 \mathrm{a}-\mathrm{f}$ & $9.25 \mathrm{a}-\mathrm{f}$ \\
\hline Caboron & $13.76 \mathrm{a}-\mathrm{g}$ & $14.34 \mathrm{a}-\mathrm{f}$ & $12.73 \mathrm{~d}-\mathrm{g}$ & $12.76 \mathrm{c}-\mathrm{h}$ & $8.58 \mathrm{~b}-\mathrm{f}$ & $7.99 \mathrm{c}-\mathrm{h}$ \\
\hline Amica & $14.03 \mathrm{a}-\mathrm{g}$ & $14.20 \mathrm{a}-\mathrm{f}$ & $11.88 \mathrm{f}-\mathrm{i}$ & $12.24 \mathrm{f}-\mathrm{i}$ & $8.33 \mathrm{~b}-\mathrm{g}$ & $8.47 \mathrm{~b}-\mathrm{f}$ \\
\hline Shetocare & $14.13 \mathrm{a}-\mathrm{f}$ & 15.21 a-e & $13.79 \mathrm{c}-\mathrm{g}$ & $17.55 \mathrm{a}-\mathrm{b}$ & $9.08 \mathrm{a}-\mathrm{f}$ & $9.89 \mathrm{a}-\mathrm{d}$ \\
\hline Hummer & $12.78 \mathrm{c}-\mathrm{h}$ & $12.92 \mathrm{c}-\mathrm{h}$ & $11.08 \mathrm{~g}-\mathrm{i}$ & $13.04 \mathrm{c}-\mathrm{i}$ & $7.42 \mathrm{~d}-\mathrm{h}$ & $7.41 \mathrm{e}-\mathrm{h}$ \\
\hline Amino $\mathrm{X}$ & $13.26 \mathrm{~b}-\mathrm{g}$ & $15.94 \mathrm{a}-\mathrm{c}$ & $15.47 \mathrm{~b}-\mathrm{e}$ & $15.48 \mathrm{~b}-\mathrm{e}$ & $8.59 b-f$ & 8.91 a-f \\
\hline Untreated & $11.86 \mathrm{c}-\mathrm{h}$ & $12.39 \mathrm{~b}-\mathrm{g}$ & $12.55 \mathrm{e}-\mathrm{i}$ & $14.35 \mathrm{c}-\mathrm{g}$ & 8.55 b-f & $9.06 \mathrm{~b}-\mathrm{f}$ \\
\hline LSD $_{0.05}$ & \multicolumn{2}{|c|}{3.481} & \multicolumn{2}{|c|}{3.139} & \multicolumn{2}{|c|}{ 2. 474} \\
\hline
\end{tabular}

Details of treatment listed in Table 3

Table 17b: Effect of the interaction between storage temperature and foliar application with commercial compounds on peroxidase and malondialdehyde content of onion seeds during growing seasons

\begin{tabular}{|c|c|c|c|c|c|c|}
\hline \multirow[t]{3}{*}{ Treatment } & \multicolumn{4}{|c|}{ Malondialdehyde content } & \multicolumn{2}{|c|}{ Peroxidase activity } \\
\hline & Room temp. & $5^{\circ} \mathrm{C}$ & Room temp. & $5^{\circ} \mathrm{C}$ & Room temp. & $5^{\circ} \mathrm{C}$ \\
\hline & \multicolumn{2}{|c|}{ Season 2008/2009 } & \multicolumn{2}{|c|}{ Season $2009 / 2010$} & \multicolumn{2}{|c|}{ Season $2009 / 2010$} \\
\hline Union $\mathrm{Zn}$ & 16.29 & 15.85 & 16.95 a-e & $14.61 \mathrm{e}$ & 7.37e-h & $7.77-\mathrm{h}$ \\
\hline Union Feer & 16.93 & 14.59 & $17.45 \mathrm{a}-\mathrm{d}$ & $16.07 \mathrm{c}-\mathrm{e}$ & $9.40 \mathrm{a}-\mathrm{e}$ & $9.46 \mathrm{a}-\mathrm{e}$ \\
\hline Union Mn & 15.92 & 13.19 & $15.93 \mathrm{c}-\mathrm{e}$ & $14.95 \mathrm{de}$ & $10.53 \mathrm{a}$ & $10.5 \mathrm{a}-\mathrm{c}$ \\
\hline Shams K & 15.50 & 14.59 & $17.83 \mathrm{a}-\mathrm{c}$ & 16.83 a-e & $8.44 \mathrm{c}-\mathrm{g}$ & $9.39 \mathrm{a}-\mathrm{e}$ \\
\hline Boron & 17.67 & 13.70 & $17.95 \mathrm{a}-\mathrm{c}$ & $14.76 \mathrm{e}$ & $9.94 a-d$ & $11.2 \mathrm{a}-\mathrm{b}$ \\
\hline Magnesium & 18.90 & 17.50 & 19.43 a & $18.76 \mathrm{a}-\mathrm{b}$ & $6.36 \mathrm{gh}$ & $7.04 \mathrm{f}-\mathrm{i}$ \\
\hline Elga 600 & 14.55 & 13.21 & $15.44 \mathrm{c}-\mathrm{e}$ & 15.16 d-e & $9.26 a-f$ & $9.96 \mathrm{a}-\mathrm{d}$ \\
\hline Caboron & 17.42 & 17.19 & $17.83 \mathrm{a}-\mathrm{c}$ & 16.59 b-e & $5.959 \mathrm{~h}$ & $6.28 \mathrm{~g}-\mathrm{h}$ \\
\hline Amica & 15.81 & 14.73 & $16.04 \mathrm{c}-\mathrm{e}$ & $15.62 \mathrm{c}-\mathrm{e}$ & $8.36 \mathrm{c}-\mathrm{g}$ & $9.35 \mathrm{a}-\mathrm{f}$ \\
\hline Shetocare & 15.62 & 14.48 & 17.23 a-e & 14.85 d-e & $10.5 \mathrm{a}-\mathrm{c}$ & $10.5 \mathrm{a}-\mathrm{c}$ \\
\hline Hummer & 16.81 & 14.50 & $16.64 \mathrm{~b}-\mathrm{e}$ & $15.67 \mathrm{c}-\mathrm{e}$ & $7.48 \mathrm{e}-\mathrm{h}$ & $8.45 \mathrm{c}-\mathrm{g}$ \\
\hline Amino $\mathrm{X}$ & 15.48 & 14.18 & 17.05 a-e & $15.78 \mathrm{c}-\mathrm{e}$ & $9.03 b-f$ & $9.99 \mathrm{a}-\mathrm{d}$ \\
\hline Untreated & 18.76 & 18.34 & $18.86 \mathrm{a}-\mathrm{b}$ & $18.74 \mathrm{a}-\mathrm{b}$ & $7.81 \mathrm{~d}-\mathrm{h}$ & $8.42 \mathrm{c}-\mathrm{g}$ \\
\hline $\mathbf{L S D}_{0.05}$ & \multicolumn{2}{|c|}{ N.S } & \multicolumn{2}{|c|}{2.650} & \multicolumn{2}{|c|}{2.333} \\
\hline
\end{tabular}

Details of treatment listed in Table 3

that the lowest value of catalase was observed for the control after 12 months of storage at both storage temperatures in both seasons. On the other hand, the highest catalase activity was recorded at the treatment with boron after 6 months of storage at $5^{\circ} \mathrm{C}$ storage temperature.

\section{Peroxidase activity}

\section{Effect of storage temperatures on peroxidase activity}

Results obtained on the effect of storage temperatures on peroxidase activity are presented in Table 15. Storage temperature had non significant effect on peroxidase activity but there was a slight increase in peroxidase activity at $5^{\circ} \mathrm{C}$ than at room temperature. These results are in agreement with those of Doijode (1990), Siegenthaler and Douet (1994), Yanping et al. (1999) and (2000) and Demirkaya et al. (2010). They found that antioxidant enzymes activity was higher in cold storage than in room temperature.

\section{Effect of storage period on peroxidase activity}

Storage period had a significant effect on peroxidase activity in both seasons. It is clear from data presented in Table 15 that the peroxidase activity was significantly decreased as storage period increased. These results are in agreement with Golovina et $\boldsymbol{a l}$. (1997) and Demirkaya et al. (2010).

\section{Effect of foliar application with commercial compounds on peroxidase activity}

Foliar application with boron had the highest peroxidase activity in both seasons as shown Table 15. This treatment had the highest peroxidase activity after harvest and had an important role as a functional, structural or regulatory factor of enzymes. These results are in agreement with those obtained by Prasad (2003) and Chatterjee $\boldsymbol{e t}$ al. (2006). 
Table 18: Effect of the interaction between storage period and foliar application with commercial compounds on catalase activity of onion seeds during $2008 / 2009$ season

\begin{tabular}{|c|c|c|c|c|c|c|}
\hline \multirow[t]{3}{*}{ Interactions } & \multicolumn{6}{|c|}{ Storage period (month) } \\
\hline & $\mathbf{0}$ & 6 & 12 & 0 & 6 & 12 \\
\hline & \multicolumn{3}{|c|}{ Season 2008 / 2009} & \multicolumn{3}{|c|}{ Season 2009 / 2010} \\
\hline Union $\mathrm{Zn}$ & 18.13 a-d & 11.76 g-1 & 8.700 o & $17.04 \mathrm{~b}-\mathrm{e}$ & $15.82 b-f$ & 9.842 k-n \\
\hline Union Feer & $19.24 \mathrm{a}-\mathrm{c}$ & $13.22 \mathrm{f}-\mathrm{j}$ & $8.963 \mathrm{j}-\mathrm{o}$ & $19.11 \mathrm{a}-\mathrm{c}$ & $12.25 \mathrm{f}-1$ & $8.037 \mathrm{~m}-\mathrm{n}$ \\
\hline Union Mn & 16.48 b-f & $11.81 \mathrm{~g}-1$ & $7.9721-0$ & $15.82 b-f$ & $11.97 \mathrm{~g}-1$ & $11.11 \mathrm{i}-\mathrm{n}$ \\
\hline Shams K & $21.36 \mathrm{a}$ & $13.59 \mathrm{e}-\mathrm{i}$ & 8.698 k-o & $21.40 \mathrm{a}$ & $14.21 \mathrm{~d}-\mathrm{i}$ & $11.95 \mathrm{~h}-1$ \\
\hline Boron & $20.16 a-b$ & 17.61 a-e & $13.11 \mathrm{f}-\mathrm{j}$ & $19.29 a-b$ & $13.79 \mathrm{~d}-\mathrm{j}$ & $17.38 \mathrm{~b}-\mathrm{d}$ \\
\hline Magnesium & $13.21 \mathrm{f}-\mathrm{j}$ & 10.19 h-m & $9.120 \mathrm{j}-\mathrm{n}$ & $14.09 \mathrm{~d}-\mathrm{j}$ & $8.7831-n$ & $7.722 \mathrm{n}$ \\
\hline Elga 600 & 13.96 d-g & 10.94 h-1 & 8.855 n-o & $15.80 \mathrm{~b}-\mathrm{g}$ & $11.46 \mathrm{i}-\mathrm{n}$ & $11.57 \mathrm{~h}-\mathrm{m}$ \\
\hline Caboron & $18.87 \mathrm{a}-\mathrm{c}$ & $13.61 \mathrm{e}-\mathrm{i}$ & 9.660 i-n & $13.67 \mathrm{~d}-\mathrm{j}$ & $12.81 \mathrm{f}-\mathrm{k}$ & $11.77 \mathrm{~h}-\mathrm{m}$ \\
\hline Amica & $15.30 \mathrm{c}-\mathrm{g}$ & 14.14 d-h & $12.92 \mathrm{f}-\mathrm{k}$ & $14.82 \mathrm{~d}-\mathrm{i}$ & 11.45 i-n & 9.903 k-n \\
\hline Shetocare & $21.43 \mathrm{a}$ & $16.50 \mathrm{~b}-\mathrm{f}$ & $6.075 \mathrm{~m}-\mathrm{o}$ & $17.10 \mathrm{~b}-\mathrm{e}$ & $15.41 \mathrm{c}-\mathrm{h}$ & $14.50 \mathrm{~d}-\mathrm{i}$ \\
\hline Hummer & $16.42 \mathrm{~b}-\mathrm{f}$ & $13.82 \mathrm{e}-\mathrm{i}$ & $8.322 \mathrm{~m}-\mathrm{o}$ & $13.56 \mathrm{~d}-\mathrm{k}$ & $12.55 \mathrm{f}-1$ & $10.08 \mathrm{j}-\mathrm{n}$ \\
\hline Amino $X$ & $19.87 a-b$ & $15.23 \mathrm{c}-\mathrm{g}$ & $8.703 \mathrm{k}-\mathrm{o}$ & $19.22 \mathrm{a}-\mathrm{c}$ & $13.67 \mathrm{~d}-\mathrm{k}$ & $13.52 \mathrm{e}-\mathrm{k}$ \\
\hline Untreated & $14.87 \mathrm{~b}-\mathrm{f}$ & $11.78 \mathrm{~g}-1$ & $6.720 \mathrm{~m}-\mathrm{o}$ & $14.15 \mathrm{~d}-\mathrm{i}$ & $12.22 \mathrm{f}-1$ & $7.99 \mathrm{n}$ \\
\hline
\end{tabular}

Details of treatment listed in Table 3

Table 19: Effect of the interaction between storage temperature, storage period and foliar application with commercial compounds on catalase activity of onion seeds during two seasons

\begin{tabular}{|c|c|c|c|c|c|c|}
\hline \multirow[t]{3}{*}{ Interactions } & \multicolumn{6}{|c|}{ Storage period (month) } \\
\hline & $\mathbf{0}$ & 6 & 12 & $\mathbf{0}$ & 6 & 12 \\
\hline & \multicolumn{3}{|c|}{ Season 2008/2009 } & \multicolumn{3}{|c|}{ Season $2009 / 2010$} \\
\hline & \multicolumn{6}{|c|}{ Room temperature } \\
\hline Union Zn & 20.13 & 12.89 & 9.927 & 19.04 & 17.87 & 9.557 \\
\hline Union Feer & 19.24 & 14.24 & 8.163 & 19.11 & 12.66 & 7.673 \\
\hline Union Mn & 16.48 & 13.04 & 6.060 & 15.82 & 11.65 & 10.36 \\
\hline Shams K & 21.36 & 14.04 & 10.75 & 21.40 & 14.14 & 11.90 \\
\hline Boron & 20.16 & 16.87 & 13.16 & 19.29 & 11.12 & 11.30 \\
\hline Magnesium & 13.21 & 9.673 & 8.877 & 14.09 & 8.340 & 7.323 \\
\hline Elga 600 & 13.96 & 10.39 & 9.033 & 15.80 & 10.58 & 10.66 \\
\hline Caboron & 18.87 & 13.26 & 9.157 & 17.34 & 13.32 & 11.21 \\
\hline Amica & 15.30 & 14.05 & 12.74 & 14.82 & 11.24 & 9.573 \\
\hline Shetocare & 21.43 & 16.06 & 9.880 & 17.10 & 12.42 & 11.85 \\
\hline Hummer & 16.42 & 14.26 & 7.667 & 13.56 & 12.37 & 7.320 \\
\hline Amino $\mathrm{X}$ & 19.87 & 14.03 & 8.877 & 19.22 & 13.97 & 13.21 \\
\hline Untreated & 16.87 & 11.59 & 5.12 & 14.15 & 11.89 & 6.62 \\
\hline \multicolumn{7}{|c|}{ Cold temperature $\left(5^{\circ} \mathrm{C}\right)$} \\
\hline Union Zn & 20.13 & 10.63 & 7.473 & 19.04 & 13.77 & 10.13 \\
\hline Union Feer & 19.24 & 12.20 & 9.763 & 19.11 & 11.84 & 8.400 \\
\hline Union Mn & 16.48 & 10.57 & 9.883 & 15.82 & 12.29 & 11.87 \\
\hline Shams K & 21.36 & 13.13 & 11.64 & 21.40 & 14.28 & 12.01 \\
\hline Boron & 20.16 & 18.35 & 13.07 & 19.29 & 16.45 & 13.46 \\
\hline Magnesium & 13.21 & 10.72 & 9.363 & 14.09 & 9.227 & 8.120 \\
\hline Elga 600 & 13.96 & 11.49 & 7.677 & 15.80 & 12.35 & 9.47 \\
\hline Caboron & 18.87 & 13.97 & 10.16 & 17.34 & 12.29 & 9.33 \\
\hline Amica & 15.30 & 14.23 & 13.09 & 14.82 & 11.67 & 10.23 \\
\hline Shetocare & 21.43 & 16.93 & 7.270 & 17.10 & 18.40 & 17.16 \\
\hline Hummer & 16.42 & 13.38 & 8.977 & 13.56 & 12.73 & 8.84 \\
\hline Amino $\mathrm{X}$ & 19.87 & 16.42 & 11.53 & 19.22 & 13.37 & 8.84 \\
\hline Untreated & 16.87 & 11.97 & 6.32 & 14.15 & 12.55 & 6.36 \\
\hline
\end{tabular}

Details of treatment listed in Table 3

\section{Effect of the interaction between storage temperature and storage period}

Regarding to the effect of the interaction between storage temperature and storage period on peroxidase activity, data in Table 20 show that peroxidase activity decreased in both storage temperatures as storage period increased in both seasons. The lowest value of peroxidase was recorded after 12 months of storage at both storage temperatures in both seasons.

\section{Effect of the interaction between storage temperature and foliar application with commercial compounds}

As shown in Table 17, data indicate that peroxidase activity decreased in all foliar application treatments as storage temperature increased. The highest value of peroxidase was recorded for boron treatment at $5^{\circ} \mathrm{C}$ in the first season, and the treatment with boron at $5^{\circ} \mathrm{C}$ and union $\mathrm{Mn}$ or shetocare at both temperatures in the second season. 
Table 20: Effect of the interaction between storage temperature and storage period on peroxidase activity of onion seeds during seasons of $2008 / 2009$ and $2009 / 2010$

\begin{tabular}{|c|c|c|c|c|}
\hline \multirow{3}{*}{$\begin{array}{l}\text { Storage period } \\
\text { (month) }\end{array}$} & \multicolumn{4}{|c|}{ Storage temperature } \\
\hline & Room temperature & Cold temp. $\left(5^{\circ} \mathrm{C}\right)$ & Room temperature & Cold temp. $\left(5^{\circ} \mathrm{C}\right)$ \\
\hline & \multicolumn{2}{|c|}{ Season 2008/2009 } & \multicolumn{2}{|c|}{ Season $2009 / 2010$} \\
\hline $\begin{array}{c}0 \\
6 \\
12 \\
\text { LSD }_{0.05}\end{array}$ & $\begin{array}{c}11.19 \mathrm{a} \\
8.513 \mathrm{~b} \\
6.020 \mathrm{c} \\
\mathbf{0 . 8 7 6}\end{array}$ & $\begin{array}{l}11.19 \mathrm{a} \\
9.214 \mathrm{~b} \\
6.098 \mathrm{c}\end{array}$ & $\begin{array}{c}10.66 \mathrm{a} \\
8.050 \mathrm{bc} \\
7.022 \mathrm{c} \\
\mathbf{1 . 2 9 5}\end{array}$ & $\begin{array}{l}10.66 \mathrm{a} \\
9.149 \mathrm{~b} \\
7.539 \mathrm{c}\end{array}$ \\
\hline
\end{tabular}

Table 21: Effect of the interaction between storage period and foliar application with commercial compounds on peroxidase activity of onion seeds during two season

\begin{tabular}{|c|c|c|c|c|c|c|}
\hline \multirow[t]{3}{*}{ Interactions } & \multicolumn{6}{|c|}{ Storage period (month) } \\
\hline & $\mathbf{0}$ & 6 & 12 & $\mathbf{0}$ & 6 & 12 \\
\hline & \multicolumn{3}{|c|}{ Season 2008 / 2009} & \multicolumn{3}{|c|}{ Season 2009 / 2010} \\
\hline Union $\mathrm{Zn}$ & $9.983 \mathrm{c}-\mathrm{g}$ & 8.942 e-k & $5.537 \mathrm{p}$ & $8.513 \mathrm{~g}-\mathrm{O}$ & 7.403 i-p & $6.812 \mathrm{k}-\mathrm{p}$ \\
\hline Union Feer & $16.68 \mathrm{a}$ & 8.392 e-m & $6.218 \mathrm{j}-\mathrm{p}$ & $11.98 \mathrm{a}-\mathrm{c}$ & $9.548 \mathrm{c}-\mathrm{k}$ & $6.775 \mathrm{k}-\mathrm{p}$ \\
\hline Union Mn & $12.56 \mathrm{~b}-\mathrm{c}$ & $10.68 \mathrm{c}-\mathrm{e}$ & $5.968 \mathrm{k}-\mathrm{p}$ & $14.28 \mathrm{a}$ & $9.955 \mathrm{c}-\mathrm{j}$ & 8.825 e-n \\
\hline Shams K & $10.41 \mathrm{c}-\mathrm{f}$ & $8.835 \mathrm{e}-1$ & $5.8371-\mathrm{p}$ & $11.30 \mathrm{~b}-\mathrm{g}$ & $9.293 \mathrm{c}-1$ & $6.165 \mathrm{n}-\mathrm{p}$ \\
\hline Boron & $12.17 \mathrm{~b}-\mathrm{d}$ & $10.75 \mathrm{~b}-\mathrm{e}$ & $9.163 \mathrm{~d}-\mathrm{j}$ & $11.71 \mathrm{a}-\mathrm{d}$ & $10.44 \mathrm{c}-\mathrm{h}$ & $9.695 \mathrm{c}-\mathrm{j}$ \\
\hline Magnesium & $6.650 \mathrm{~h}-\mathrm{o}$ & $6.228 \mathrm{j}-\mathrm{p}$ & $4.680 \mathrm{n}-\mathrm{p}$ & 7.193 j-p & $6.5321-p$ & $6.388 \mathrm{~m}-\mathrm{p}$ \\
\hline Elga 600 & $13.71 \mathrm{a}-\mathrm{b}$ & $6.738 \mathrm{~h}-\mathrm{O}$ & $7.640 \mathrm{f}-\mathrm{n}$ & $14.00 \mathrm{a}-\mathrm{b}$ & $8.122 \mathrm{~h}-\mathrm{o}$ & $6.723 \mathrm{k}-\mathrm{p}$ \\
\hline Caboron & $10.55 \mathrm{c}-\mathrm{f}$ & $9.808 \mathrm{c}-\mathrm{g}$ & 4.512 o-p & 7.267 j-p & 6.157 n-p & $4.937 \mathrm{p}$ \\
\hline Amica & $9.507 \mathrm{~b}-\mathrm{h}$ & $9.355 \mathrm{~d}-\mathrm{i}$ & $6.350 \mathrm{i}-\mathrm{p}$ & $10.15 \mathrm{c}-\mathrm{i}$ & $9.048 \mathrm{~d}-\mathrm{m}$ & 7.383 i-p \\
\hline Shetocare & $11.99 \mathrm{~b}-\mathrm{d}$ & $9.523 \mathrm{~d}-\mathrm{h}$ & $6.963 \mathrm{~g}-\mathrm{o}$ & 11.66 a-e & $10.55 \mathrm{c}-\mathrm{h}$ & $9.437 \mathrm{c}-\mathrm{k}$ \\
\hline Hummer & 8.790 e-1 & 7.848 e-m & $5.630 \mathrm{~m}-\mathrm{p}$ & 8.627 g-n & 8.182 h-o & 7.108 j-p \\
\hline Amino $\mathrm{X}$ & $10.38 \mathrm{c}-\mathrm{f}$ & $9.355 \mathrm{~d}-\mathrm{i}$ & 6.528 h-p & $10.36 \mathrm{c}-\mathrm{h}$ & $9.455 \mathrm{c}-\mathrm{k}$ & $8.723 \mathrm{f}-\mathrm{n}$ \\
\hline Untreated & $12.06 \mathrm{~b}-\mathrm{d}$ & $8.775 \mathrm{e}-1$ & $5.740 \mathrm{~m}-\mathrm{p}$ & $11.56 \mathrm{a}-\mathrm{f}$ & $7.115 \mathrm{j}-\mathrm{p}$ & 5.677 o-p \\
\hline $\mathbf{L S D}_{0.05}$ & \multicolumn{3}{|c|}{3.030} & \multicolumn{3}{|c|}{2.857} \\
\hline
\end{tabular}

Details of treatment listed in Table 3

\section{Effect of the interaction between storage period and foliar application with commercial compounds}

It is clear from data presented in Table 21 that peroxidase activity was decreased in all foliar application treatments as storage period increased. The highest value of peroxidase activity after 12 months of storage was recorded for boron treatment in the first season, and boron or shetocare treatments in the second season.

\section{Effect of the interaction between storage temperature, storage period and foliar application with commercial products}

Regarding the of interaction between storage temperature, storage period and foliar application with commercial compounds on peroxidase activity, data in Table 22 show that the highest value of peroxidase was observed in the treatment with union $\mathrm{Mn}$ or boron at room temperature and shams $\mathrm{K}$ or boron at $5^{\circ} \mathrm{C}$ in the first season. Meanwhile, the treatment with union $\mathrm{Mn}$, boron or shetocare at room temperature and the treatment with boron, shetocare or amino $\mathrm{X}$ at $5^{\circ} \mathrm{C}$ had the highest peroxidase value in the second season.

\section{Malondialdehyde content}

\section{Effect of storage temperature on malondialdehyde content}

Storage temperature had non significant effect on malondialdehyde content in the first season, but there was significant effect of storage temperature on malondialdehyde content in the second season. Seed stored at room temperature had the higher malondialdehyde content than which stored at $5^{\circ} \mathrm{C}$ (Table 23). These results may be due to that room temperature had the lower catalase and peroxidase activity than $5^{\circ} \mathrm{C}$. These results are in agreement with those obtained by Yanping $\boldsymbol{e t}$ al. (2000) and Demirkaya et al. (2010). They found that as storage temperature increased the malondialdehyde content increased and antioxidant activity decreased.

\section{Effect of storage period on malondialdehyde content}

Regarding the effect of storage period on malondialdehyde content, data in Table $\mathbf{2 3}$ show that malondialdehyde content increased as storage period increased. These results are in agreement with those obtained by Golovina $\boldsymbol{e t}$ al . (1997) and Demrikaya et al. (2010). They reported that the malondialdehyde content increased as storage period increased.

\section{Effect of foliar application with commercial compounds on malondialdehyde content}

Concerning the effect of foliar application with commercial compounds on malondialdehyde content, data in Table 23 show that the treatment with magnesium, caboron and the control had the highest malondialdehyde content in the first season, and the treatment with magnesium and the untreated in the second season. These treatments had the lowest antioxidant activity. These results may be due to the fact that there was a negative correlation between antioxidant activity and malondialdehyde content (Xin and Wang, 2006). 
Table 22: Effect of the interaction between storage temperature, storage period and foliar application with commercial compounds on peroxidase activity of onion seeds during seasons of 2008/2009 and 2009/2010

\begin{tabular}{|c|c|c|c|c|c|c|}
\hline \multirow[t]{3}{*}{ Interactions } & \multicolumn{6}{|c|}{ Storage period (month) } \\
\hline & $\mathbf{0}$ & 6 & 12 & $\mathbf{0}$ & 6 & 12 \\
\hline & \multicolumn{3}{|c|}{ Season 2008/2009 } & \multicolumn{3}{|c|}{ Season $2009 / 2010$} \\
\hline & \multicolumn{6}{|c|}{ Room temperature } \\
\hline Union $\mathrm{Zn}$ & 9.983 & 8.557 & 6.540 & 8.513 & 7.080 & 6.543 \\
\hline Union Feer & 16.68 & 8.180 & 6.187 & 11.98 & 8.970 & 7.277 \\
\hline Union Mn & 12.56 & 11.70 & 9.507 & 14.28 & 10.72 & 9.622 \\
\hline Shams K & 17.01 & 8.490 & 4.817 & 11.30 & 8.357 & 5.667 \\
\hline Boron & 12.17 & 10.53 & 8.090 & 11.71 & 9.293 & 8.827 \\
\hline Magnesium & 6.650 & 5.887 & 4.983 & 7.193 & 6.367 & 5.547 \\
\hline Elga 600 & 13.71 & 7.280 & 7.423 & 14.00 & 7.633 & 6.157 \\
\hline Caboron & 10.55 & 8.980 & 6.217 & 7.267 & 6.123 & 4.487 \\
\hline Amica & 9.507 & 8.910 & 6.590 & 10.15 & 7.737 & 7.217 \\
\hline Shetocare & 11.99 & 8.143 & 7.133 & 11.66 & 10.26 & 9.637 \\
\hline Hummer & 8.790 & 7.623 & 5.867 & 8.627 & 7.367 & 6.463 \\
\hline Amino $\mathrm{X}$ & 10.38 & 8.793 & 6.610 & 10.36 & 9.000 & 7.733 \\
\hline Untreated & 12.06 & 7.597 & 5.297 & 11.56 & 6.867 & 5.017 \\
\hline \multicolumn{7}{|c|}{ Cold temperature $\left(5^{\circ} \mathrm{C}\right)$} \\
\hline Union Zn & 9.983 & 9.327 & 7.533 & 8.513 & 7.727 & 7.080 \\
\hline Union Feer & 16.68 & 8.603 & 6.250 & 11.98 & 10.13 & 6.273 \\
\hline Union Mn & 12.56 & 9.653 & 6.430 & 14.28 & 10.31 & 6.933 \\
\hline Shams K & 17.01 & 9.180 & 6.857 & 11.30 & 10.23 & 6.663 \\
\hline Boron & 12.17 & 10.97 & 10.24 & 11.71 & 11.59 & 10.56 \\
\hline Magnesium & 6.650 & 6.570 & 5.377 & 7.193 & 6.697 & 5.230 \\
\hline Elga 600 & 13.71 & 6.197 & 7.857 & 14.00 & 8.610 & 7.290 \\
\hline Caboron & 10.55 & 10.64 & 6.807 & 7.267 & 6.190 & 5.387 \\
\hline Amica & 9.507 & 9.800 & 6.110 & 10.15 & 10.36 & 7.550 \\
\hline Shetocare & 11.99 & 10.90 & 6.793 & 11.66 & 10.83 & 9.237 \\
\hline Hummer & 8.790 & 8.073 & 5.393 & 8.627 & 8.997 & 7.753 \\
\hline Amino $\mathrm{X}$ & 10.38 & 9.917 & 6.447 & 10.36 & 9.910 & 9.713 \\
\hline Untreated & 12.06 & 9.953 & 5.183 & 11.56 & 7.363 & 4.337 \\
\hline
\end{tabular}

Details of treatment listed in Table 3

Table 23: Effect of storage temperature, storage period and foliar application with commercial compounds on malondialdehyde content of onion seeds during seasons of 2008 / 2009 and 2009/2010

\begin{tabular}{|c|c|c|}
\hline \multirow[t]{2}{*}{ Treatments } & \multicolumn{2}{|c|}{ Malondialdehyde (mmol $\mathrm{g}^{-1}$ fw) } \\
\hline & $2008 / 2009$ & $2009 / 2010$ \\
\hline $\begin{array}{l}\text { Storage temperature } \\
\text { Room temperature } \\
\text { Cold temperature }\left(5^{\circ} \mathrm{C}\right) \\
\text { LSD }_{0.05}\end{array}$ & $\begin{array}{l}16.44 \\
16.00 \\
\text { N.S. }\end{array}$ & $\begin{array}{c}17.28 \mathrm{a} \\
16.03 \mathrm{~b} \\
0.887\end{array}$ \\
\hline $\begin{array}{c}\text { Storage periods (month) } \\
0 \\
6 \\
12 \\
\text { LSD }_{0.05}\end{array}$ & $\begin{array}{c}12.02 \mathrm{c} \\
16.17 \mathrm{~b} \\
20.46 \mathrm{a} \\
2.934\end{array}$ & $\begin{array}{c}12.81 \mathrm{c} \\
17.00 \mathrm{~b} \\
20.15 \mathrm{a} \\
0.650\end{array}$ \\
\hline $\begin{array}{l}\text { Foliar application } \\
\text { Union } \mathrm{Zn} \\
\text { Union Feer } \\
\text { Union } \mathrm{Mn} \\
\text { Shams } \mathrm{K} \\
\text { Boron } \\
\text { Magnesium } \\
\text { Elga } 600 \\
\text { Caboron } \\
\text { Amica } \\
\text { Shetocare } \\
\text { Hummer } \\
\text { Amino X } \\
\text { Untreated } \\
\text { LSD } \\
\text { 0.05 }\end{array}$ & $\begin{array}{l}15.07 \mathrm{~b} \\
15.76 \mathrm{~b} \\
14.55 \mathrm{~b} \\
15.05 \mathrm{~b} \\
15.68 \mathrm{~b} \\
18.20 \mathrm{a} \\
13.88 \mathrm{~b} \\
16.80 \mathrm{a} \\
15.27 \mathrm{~b} \\
15.05 \mathrm{~b} \\
15.66 \mathrm{~b} \\
14.83 \mathrm{~b} \\
18.05 \mathrm{a} \\
\mathbf{2 . 8 1}\end{array}$ & $\begin{array}{l}15.78 \mathrm{c}-\mathrm{e} \\
16.76 \mathrm{c}-\mathrm{e} \\
15.44 \mathrm{~d}-\mathrm{e} \\
17.33 \mathrm{a}-\mathrm{c} \\
16.36 \mathrm{c}-\mathrm{e} \\
19.09 \mathrm{a} \\
15.30 \mathrm{e} \\
17.21 \mathrm{~b}-\mathrm{d} \\
15.83 \mathrm{c}-\mathrm{e} \\
16.04 \mathrm{c}-\mathrm{e} \\
16.16 \mathrm{c}-\mathrm{e} \\
16.42 \mathrm{c}-\mathrm{e} \\
18.80 \mathrm{a}-\mathrm{b} \\
\mathbf{1 . 8 7 4}\end{array}$ \\
\hline
\end{tabular}

Details of treatment listed in Table 3 
Table 24: Effect of the interaction between storage temperature and storage period on malondialdehyde content of onion seeds during seasons of 2008/2009 and $2009 / 2010$

\begin{tabular}{|c|c|c|c|c|}
\hline \multirow{3}{*}{$\begin{array}{l}\text { Storage period } \\
\text { (month) }\end{array}$} & \multicolumn{4}{|c|}{ Storage temperature } \\
\hline & Room temperature & Cold temp. $\left(5^{\circ} \mathrm{C}\right)$ & Room temperature & Cold temp. $\left(5^{\circ} \mathrm{C}\right)$ \\
\hline & \multicolumn{2}{|c|}{ Season $2008 / 2009$} & \multicolumn{2}{|c|}{ Season $2009 / 2010$} \\
\hline $\mathbf{0}$ & \multirow{4}{*}{$\begin{array}{c}12.02 \mathrm{c} \\
17.19 \mathrm{ab} \\
20.83 \mathrm{a} \\
4.149\end{array}$} & \multirow{4}{*}{$\begin{array}{c}12.02 \mathrm{c} \\
15.16 \mathrm{bc} \\
20.09 \mathrm{a}\end{array}$} & \multirow{4}{*}{$\begin{array}{c}12.81 \mathrm{e} \\
18.13 \mathrm{c} \\
20.89 \mathrm{a} \\
0.9200\end{array}$} & \multirow{4}{*}{$\begin{array}{l}12.81 \mathrm{e} \\
15.87 \mathrm{~d} \\
19.41 \mathrm{~b}\end{array}$} \\
\hline 6 & & & & \\
\hline 12 & & & & \\
\hline $\mathbf{L S D}_{0.05}$ & & & & \\
\hline
\end{tabular}

Table 25: Effect of the interaction between storage period and foliar application with commercial compounds on malondialdehyde content of onion seeds during two seasons

\begin{tabular}{|c|c|c|c|c|c|c|}
\hline \multirow[t]{3}{*}{ Treatments } & \multicolumn{6}{|c|}{ Storage period (month) } \\
\hline & $\mathbf{0}$ & 6 & 12 & $\mathbf{0}$ & 6 & 12 \\
\hline & \multicolumn{3}{|c|}{ Season 2008/2009 } & \multicolumn{3}{|c|}{ Season $2009 / 2010$} \\
\hline Union $\mathrm{Zn}$ & $10.08 \mathrm{c}-\mathrm{e}$ & $16.23 \mathrm{~b}-\mathrm{e}$ & $19.91 \mathrm{a}-\mathrm{c}$ & $11.11 \mathrm{~m}-\mathrm{o}$ & $16.95 \mathrm{di}$ & 19.28 a-f \\
\hline Union Feer & $12.66 \mathrm{~b}-\mathrm{e}$ & $16.41 \mathrm{~b}-\mathrm{e}$ & $18.22 \mathrm{~b}-\mathrm{e}$ & 12.71 lo & $16.64 \mathrm{ei}$ & 20.93 a \\
\hline Union Mn & $9.353 \mathrm{~d}-\mathrm{e}$ & $16.20 \mathrm{~b}-\mathrm{e}$ & $18.11 \mathrm{~b}-\mathrm{e}$ & 9.920 no & $17.23 \mathrm{ci}$ & $19.17 \mathrm{a}-\mathrm{g}$ \\
\hline Shams K & $12.71 \mathrm{~b}-\mathrm{e}$ & $14.88 \mathrm{~b}-\mathrm{e}$ & $17.55 \mathrm{~b}-\mathrm{e}$ & $14.94 \mathrm{hl}$ & $16.33 \mathrm{fj}$ & $20.72 \mathrm{a}$ \\
\hline Boron & $8.840 \mathrm{e}$ & $13.09 \mathrm{~b}-\mathrm{e}$ & $19.12 \mathrm{~b}-\mathrm{d}$ & 9.667 o & $15.04 \mathrm{hl}$ & 19.36 a-f \\
\hline Magnesium & $15.45 \mathrm{~b}-\mathrm{e}$ & $18.50 \mathrm{~b}-\mathrm{e}$ & $20.64 \mathrm{a}$ & $15.97 \mathrm{gk}$ & 20.13 ad & $21.19 \mathrm{a}$ \\
\hline Elga 600 & $8.527 \mathrm{e}$ & $15.38 \mathrm{~b}-\mathrm{e}$ & $17.75 \mathrm{~b}-\mathrm{e}$ & $9.820 \mathrm{o}$ & $15.48 \mathrm{hl}$ & $20.59 \mathrm{a}-\mathrm{b}$ \\
\hline Caboron & $14.11 \mathrm{~b}-\mathrm{e}$ & $16.84 \mathrm{~b}-\mathrm{e}$ & $19.46 \mathrm{a}-\mathrm{c}$ & 13.13 jn & $18.99 \mathrm{ag}$ & 19.51 a-f \\
\hline Amica & $12.76 \mathrm{~b}-\mathrm{e}$ & $14.06 \mathrm{~b}-\mathrm{e}$ & $18.99 \mathrm{~b}-\mathrm{d}$ & 12.71 lo & $14.44 \mathrm{hl}$ & $20.33 \mathrm{a}-\mathrm{c}$ \\
\hline Shetocare & 12.92 b-e & $14.10 \mathrm{~b}-\mathrm{e}$ & $18.14 \mathrm{~b}-\mathrm{e}$ & $14.11 \mathrm{im}$ & $13.10 \mathrm{jn}$ & $20.90 \mathrm{a}$ \\
\hline Hummer & $13.02 \mathrm{~b}-\mathrm{e}$ & 14.44 b-e & $19.51 \mathrm{~b}-\mathrm{c}$ & $14.11 \mathrm{im}$ & $15.35 \mathrm{hl}$ & $19.01 \mathrm{a}-\mathrm{g}$ \\
\hline Amino $X$ & $10.85 \mathrm{~b}-\mathrm{e}$ & $15.06 \mathrm{~b}-\mathrm{e}$ & $18.58 \mathrm{~b}-\mathrm{e}$ & $12.82 \mathrm{ko}$ & $17.44 \mathrm{bh}$ & $18.99 \mathrm{a}-\mathrm{g}$ \\
\hline Untreated & 15.04 b-e & $19.09 \mathrm{bd}$ & $20.02 \mathrm{a}-\mathrm{c}$ & $15.55 \mathrm{hl}$ & $19.87 \mathrm{ae}$ & $20.98 \mathrm{a}$ \\
\hline LSD $_{0.05}$ & \multicolumn{3}{|c|}{10.06} & \multicolumn{3}{|c|}{3.246} \\
\hline
\end{tabular}

Details of treatment listed in Table 3

\section{Effect of interaction between storage temperature and storage period}

With respect to the effect of the interaction between storage temperature and storage period on malondialdehyde content, data in Table $\mathbf{2 4}$ show that malondialdehyde content increased as storage period increased at both storage temperatures in both seasons. The highest malondialdehyde content was recorded after 12 months of storage at both storage temperatures in the first season, and at room temperature in the second season.

\section{Effect of the interaction between storage temperature and foliar application with commercial compounds}

Regarding the effect of the interaction between storage temperatures and foliar application, data in Table $\mathbf{1 7}$ indicate that the malondialdehyde content at room temperature is higher than that at $5^{\circ} \mathrm{C}$ in all tested treatments in both seasons. The statistical analysis showed non significant effect of the interaction between storage temperature and foliar spray treatments on malondialdehyde content in the first season. The highest malondialdehyde content was recorded for magnesium and the untreated at both storage temperatures in the second season.

\section{Effect of the interaction between storage period and foliar application with commercial compounds}

As shown in Table 25 data indicate that malondialdehyde content increased as storage period increased in all tested treatments in both seasons. The highest value of the malondialdehyde was recorded after 12 months of storage for union $\mathrm{Zn}$, magnesium, caboron and the control in the first season, the control showed the highest malondialdehyde content after 6 months of storage. Regarding the effect of the interaction between storage period and foliar spraying at 12 months of storage, data showed that all tested treatments had the highest malondialdehyde content.

\section{Effect of the interaction between storage temperature, storage period and foliar application with commercial compounds}

The interaction between storage temperature, storage period and foliar application with commercial compounds on malondialdehyde content was presented in Table 26. The highest malondialdehyde content was recorded for magnesium, hummer and the control after 12 months of storage at room temperature in the first season, and for treatment with union feer, shams K, magnesium, elga 600, amica or shetocare at room temperature and the untreated at both storage temperatures in the second season. It could be summarized that foliar spraying of onion plants by magnesium or by water (untreated treatment) gave the highest malondialdehyde content and the lowest catalase and peroxidase activity of onion seed.

Nitrogen and sulfur availability affect growth, yield, flavor and quality of onion. Potassium plays an important role in promotion of enzymes activity and enhancing the translocation 
Table 26: Effect of the interaction between storage temperature, storage period and foliar application with commercial compounds on malondialdehyde content of onion seeds during seasons of 2008/2009 and 2009/2010

\begin{tabular}{|c|c|c|c|c|c|c|}
\hline \multirow[t]{3}{*}{ Interactions } & \multicolumn{6}{|c|}{ Storage period (month) } \\
\hline & $\mathbf{0}$ & 6 & 12 & $\mathbf{0}$ & 6 & 12 \\
\hline & \multicolumn{3}{|c|}{ Season $2008 / 2009$} & \multicolumn{3}{|c|}{ Season 2009/2010 } \\
\hline & \multicolumn{6}{|c|}{ Room temperature } \\
\hline Union Zn & 10.08 & 19.27 & 19.53 & 11.11 & 20.05 & 19.69 \\
\hline Union Feer & 12.66 & 18.81 & 19.33 & 12.71 & 17.88 & 21.75 \\
\hline Union Mn & 9.353 & 18.34 & 20.05 & 9.920 & 18.65 & 19.22 \\
\hline Shams K & 12.71 & 15.29 & 18.50 & 14.94 & 17.15 & 21.39 \\
\hline Boron & 8.840 & 15.75 & 18.43 & 9.667 & 21.65 & 20.53 \\
\hline Magnesium & 15.45 & 19.22 & 22.01 & 15.97 & 20.46 & 21.86 \\
\hline Elga 600 & 8.527 & 16.23 & 18.91 & 9.820 & 15.45 & 21.03 \\
\hline Caboron & 14.11 & 15.97 & 19.17 & 13.13 & 19.64 & 20.72 \\
\hline Amica & 12.76 & 14.31 & 20.36 & 12.71 & 14.68 & 20.72 \\
\hline Shetocare & 12.92 & 15.40 & 18.55 & 14.11 & 15.92 & 21.65 \\
\hline Hummer & 13.02 & 14.62 & 22.79 & 14.11 & 15.55 & 20.26 \\
\hline Amino $\mathrm{X}$ & 10.85 & 16.02 & 19.58 & 12.82 & 18.65 & 19.69 \\
\hline Untreated & 15.04 & 18.24 & 21.00 & 15.55 & 19.94 & 21.08 \\
\hline \multicolumn{7}{|c|}{ Cold temperature $\left(5^{\circ} \mathrm{C}\right)$} \\
\hline Union Zn & 10.08 & 13.18 & 16.87 & 11.11 & 13.85 & 18.86 \\
\hline Union Feer & 12.66 & 14.00 & 17.10 & 12.71 & 15.40 & 20.10 \\
\hline Union Mn & 9.353 & 14.05 & 16.17 & 9.920 & 15.81 & 19.12 \\
\hline Shams K & 12.71 & 14.47 & 16.59 & 14.94 & 15.50 & 20.05 \\
\hline Boron & 8.840 & 16.43 & 15.81 & 9.667 & 16.43 & 18.19 \\
\hline Magnesium & 15.45 & 17.78 & 19.27 & 15.97 & 19.79 & 20.52 \\
\hline Elga 600 & 8.527 & 14.52 & 16.59 & 9.820 & 15.50 & 20.15 \\
\hline Caboron & 14.11 & 17.72 & 19.74 & 13.13 & 18.34 & 18.29 \\
\hline Amica & 12.76 & 13.80 & 17.62 & 12.71 & 14.21 & 19.94 \\
\hline Shetocare & 12.92 & 12.79 & 17.73 & 10.11 & 14.28 & 20.15 \\
\hline Hummer & 13.02 & 14.26 & 16.23 & 14.11 & 15.14 & 17.77 \\
\hline Amino $\mathrm{X}$ & 10.85 & 14.11 & 17.57 & 12.82 & 16.23 & 18.29 \\
\hline Untreated & 15.04 & 19.95 & 20.05 & 15.55 & 19.79 & 20.87 \\
\hline
\end{tabular}

Details of treatment listed in Table 3

of assimilates. K deficiency dramatically reduces dry matter accumulation and affects assimilate partitioning among plant tissues. Onion pungency was affected by application of high levels of nitrogen; it can be attributed to the role of nitrogen in the amino acids and pyruvic acid production. Significant variation in the pyruvic acid content among clones under the high $S$ nutrition reported by Hamilton et al. (1997); however under the low S treatment, it was not significant. There is a positive correlation between the amount of vitamin $\mathrm{C}$ and sulfur availability. However, increasing the amount of nitrogen availability may reduce the amount of vitamin $\mathrm{C}$. Application of $\mathrm{K}_{2} \mathrm{SO}_{4}$ fertilizer affects accumulation of total soluble solids in onion (El-Bassiony, 2006). Increase in sulfur dose tended to decrease dry matter accumulation (Nasreen et al. 2003). Abd El-Al et al. (2005) found that increasing of the soil potassium content had a significant effect on growth characters of onion (Bolandnazar et al. 2012).

\section{Conclusion}

Onion has great importance in the diet of Iranian people. Because, onion has vitamins such as A, B1, B2, C, nicotinic acid, pantothenic acid and important substances such as protein, calcium, phosphorus, potassium and traces of $\mathrm{Fe}$, $\mathrm{Al}, \mathrm{Cu}, \mathrm{Zn}, \mathrm{Mn}$ and I. Moreover, it has anti-fungal and anti- bacterial properties. Onion contains an acrid volatile oil with a pungent smell. Researchers showed that onions with higher pungency have better capacity to prevent tumor growth and also, it can protect against heart attack. It has been shown that, using of sulfur could increase onion pungency. Both pungency and volatile $S$ in onions were increased in response to increasing the elemental $\mathrm{S}$ applications.

Storage in $5^{\circ} \mathrm{C}$ had the higher germination percentage than storage in room temperature. Results indicated that as storage period increased the germination percentage decreased. The treatment with boron or amica in the first season had the highest germination percentage. While, the treatment with union $\mathrm{Zn}$, union feer, union $\mathrm{Mn}$, boron, elga 600 , caboron, amica, hummer or amino $\mathrm{X}$ had the highest germination percentage in the second season. Storage in $5^{\circ} \mathrm{C}$ resulted in higher moisture content than storage in room temperature. Regarding the effect of storage period on moisture content, the water content was significantly increased with prolongation of storage period. The lowest values of water content were recorded for treatments with union feer, shams $\mathrm{K}$ or boron in the first season, and union feer, shams K, boron, magnesium, shetocare or hummer in the second one. The results showed that there was non significant effect of storage temperature on catalase activity in both seasons, but there was a slight increase in catalase activity in seed stored in $5^{\circ} \mathrm{C}$ than in room temperature. 
Catalase activity was significantly decreased as storage period increased. The treatment with shams $\mathrm{K}$, boron, shetocare or amino $\mathrm{X}$ had the highest catalase activity in both seasons. Storage temperature had non significant effect on peroxidase activity, but there was a slight increase in peroxidase activity in $5^{\circ} \mathrm{C}$ than in room temperature. Peroxidase activity was significantly decreased as storage period increased. Foliar application with boron had the highest peroxidase activity in both seasons. Seed stored in room temperature had the higher malondialdehyde content than those stored in $5^{\circ} \mathrm{C}$ in the second season. The malondialdehyde content increased as storage period increased. The treatment with magnesium, caboron and the control in the first season, and the treatment with magnesium and the control in the second season had the highest malondialdehyde content.

\section{References}

Abd El-Al, F.S.; M. R. Shafeek A. A. Ahmed; A. M. Shaheen (2005). Response of growth and yield of onion plants to potassium fertilizer and humic acid. J. Agric. Sci. Mansoura Univ. 30, 315326.

Abd El-Gawad, K. F. (2012). Effect of some pre- and postharvest treatments on onion seed production and longevity. Ph. D. Thesis, Cairo Uni., Faculty of Agriculture, Vegetable Crops Dept., Egypt.

Ahn, J.-J.; G.-R. Kim; K. Akram; K.-S. Kim; J.-H. Kwon (2012). Luminescence characteristics of minerals separated from irradiated onions during storage under different light conditions. Radiation Physics and Chemistry, Vol. 81: 1215-1219.

Amako, A.; K. Chen; K. Asada (1994). Separate assays specific for ascorbate peroxidase and guaiacol peroxidase and for the chloroplastic and cytosilic isoenzymes of ascorbate peroxidase in plant. Plant Cell Physiology, 35: 497-504.

Ayala-Zavala, J. F.; S. Y. Wang; C. Y. Wang; G. A. GonzalezAguilar (2004). Effect of storage temperatures on antioxidant capacity and aroma compounds in strawberry fruit. Lebensm.-Wiss. -Technol., 37: 687-695.

Bailly, C., A. Benamar; F. Corizineau; D. Come (1996). Changes in malondialdehyde content and in superoxide dismutase, catalase and glutathione reductase activities in sunflower seeds as related to deterioration during accelerated ageing. Physiol. Plant. 97: 104110

Beaudry, R. M. (2000). Responses of Horticultural Commodities to Low Oxygen: Limits to the Expanded Use of Modified Atmosphere Packaging. Hort Technology, Vol. July-September 2000 10(3): 491 -500 .

Bendegumbal, C. S. (2007). Studies on effect of organics on seed yield and quality in onion (Allium cepa L.) cv. N-53. M. Sc. Thesis. Department of Seed and Technology, College of Agriculture, University of Agricultural Sciences, Dharwad- 580 005, India.

Benkeblia, N. (2000). Phenylalanine ammonia-lyase, peroxidase, pyruvic acid and total phenolics variations in onion bulbs during long-term storage. Food Sci. Technol. Leb. 33: 112-116.

Benkeblia, N.; S. Onodera; N. Shiomi (2005). Variation in 1-fructoexohydrolase (1-FEH) and 1-kestose-hydrolysing (1-KH) activities and fructo-oligosaccharide (FOS) status in onion bulbs. Influence of temperature and storage time. J. Sci. Food Agric. 85: 227-234
Bolandnazar, S.; M. Mollavali; S. J. Tabatabaei (2012). Influence of $\mathrm{NH}_{4} \mathrm{NO}_{3}$ and $\mathrm{K}_{2} \mathrm{SO}_{4}$ on qualitative characteristics of onion. Scientia Horticulturae, 136: 24-28.

Calvin, L.; B. Avendano; R. Schwentesius (2004). The economics of food safety: the case of green onions and Hepatitis A outbreaks. Econ. Res. Ser., USDA /http:// www.ers.usda.gov/publications/vgs/ nov04/vgs30501/vgs30501.pdfS.

Chance, B.; A. C. Maehly (1955). Assay of catalase and peroxidase. Methods in Enzymology, 2: 764-775.

Chatterjee, C.; G. Rajeev; B. K. Dube (2006). Impact of iron stress on biomass, yield, metabolism and quality of potato (Solanum tuberosum L.). Scientia Horticulturae, 108: 1- 6.

Chope, G. A.; L. A. Terry; P. J. White (2006). Effect of controlled atmosphere storage on abscisic acid concentration and other biochemical attributes of onion bulbs. Postharvest Biology and Technology, Vol. 39: 233-242.

Deluche, J. C.; C. C. Baskin (1973). Accelerated ageing techniques for predicting the relative storability of seed lots. Seed Sci. Technol., 1: 427-452.

Demirkaya, M.; K. J. Dietz, H. Ö. Sivritepe (2010). Changes in Antioxidant Enzymes during Ageing of Onion Seeds. Not. Bot. Hort. Agrobot. Cluj, Vol. 38 (1): 49-52.

Doijode, S. D. (1990). The influence of storage conditions on germination of onion seeds. Journal of Maharashtra Agricultural Universities, 15(1): 34-35. Cited from CAB Abstracts.

El-Bassiony, A. M. (2006). Effect of potassium fertilization on growth, yield and quality of onion plants. J. Appl. Sci. Res. 2: 780785

FAO (2012). Major food and agricultural commodities and producers - Fao.org. http://faostat.fao.org/site/567/DesktopDefault. aspx?PageID=567\#ancor Retrieved 2012-07-22.

Fonseca, S. C.; F. A. R. Oliveira; J. K. Brecht (2002). Modelling respiration rate of fresh fruits and vegetables for modified atmosphere packages: a review. Journal of Food Engineering, Vol. 52: 99-119.

Fujikura, Y.; C. M. Karssen (1995). Molecular studies on osmoprimed seeds of cauliflower, a partial amino acid sequence of a vigour-related protein and osmopriming-enhanced expression of putative aspartic protease. Seed Sci. Res. 5: 177-181.

Garg, M. K.; C. Pitam (2005). Effect of packaging material and storage place on storability of seeds a simulation study. Seed Research, 33 (1): 57-60.

Gaviola, J.; A. Ordovini; R. Lepez; M. A. Makuch (2006). Evolution of onion seed quality stored in non-controlled conditions. Agricultura Tecnica, 66(1): 13-20. Cited from CAB Abstracts.

Golovina, E. A.; W. F. Wolkers; F. A. Hoekstra (1997). Long-term stability of protein secondary structure in dry seeds. Comparative Biochemistry and Physiology, 117(3): 343-348. 10-15 September 1995

Gorris, L.; B. Tauscher (1999). Quality and safety aspects of novel minimal processing technology. In: F. A. R. Oliveira, \& J. C. Oliveira (Eds.). Processing of foods: Quality optimization and process assessment (pp. 325-339). Boca Raton, USA: CRC Press.

Hamilton, B. K.; L. M. Pike; K. S. Yoo (1997). Clonal variations of pungency, sugar content, and bulb weight of onions due to sulfur nutrition. Sci. Hortic. 71: 131-136.

Health, R. L.; L.Parker (1968). Peroxidation in isolated chloroplasts. 1. Kinetics and stoichiometry of fatty acid peroxidation. Arch. Biochem. Biophys., 125: 189-198. 
Heidari, M.; P. Jamashidi (2011). Effects of salinity and potassium application on antioxidant enzymes activities and physiological parameters in pearl millet. Agricultural Sciences in China, 10(2): 228-237.

Ilbi, H.; B. Eser (2002). The effects of pre-storage treatments on ageing in onion seeds. Acta Horticulturae, No. 579: 613-618.

Kavak, S.; B. Eser (2009). Influence of polymer coating on water uptake and germination of onion (Allium cepa L. cv. Aki) seeds before and after storage. Scientia Horticulturae, No. 121: 7-11.

Kevers, C., M. Falkowski; J. Tabart; J.-O. Defraigne; J. Dommes; J. Pincemail (2007). Evolution of antioxidant capacity during storage of selected fruits and vegetables. Journal of Agricultural and Food Chemistry, Vol. 55(21): 8596-8603.

Khokhar, K. M. (2009). Effect of set-size and storage temperature on bolting, bulbing and seed yield in two onion cultivars. Scientia Horticulturae, Vol. 122: 187-194.

Khokhar, K. M.; P. Hadley; S. Pearson (2007). Effect of cold temperature durations of onion sets in store on the incidence of bolting, bulbing and seed yield. Sci. Hortic. 112: 16-22.

Lazarenko, L. M.; V. F. Bezrukov (2008). Dynamics of chromosomal instability in welsh onion (Allium fistulosum L.): influence of seed storage temperature. Cytology and Genetics, 42 (5): 335-341

Lu, X.; J. Wang; H. M. Al-Qadiri; C. F. Ross; J. R. Powers; J. Tang; B. A. Rasco (2011). Determination of total phenolic content and antioxidant capacity of onion (Allium cepa) and shallot (Allium oschaninii) using infrared spectroscopy. Food Chemistry, Vol. 129: 637-644.

McDonald, M. B. (1999). Seed deterioration: physiology, repair and assessment. Seed Sci. Technol. 27: 177-237.

Muhammad, A.; M. A. Anjum (2002). Evaluation of physiological quality of onion seed stored for different periods. International Journal of Agriculture and Biology, 4(3): 365-369.

Nasreen, S., S. M. Imamul Haq, M. Altab Hossain (2003). Sulfur effects on growth responses and yield of onion. Asian J. Plant Sci. 2: 897-2002.

Navarro, J. M.; P. Flores; C. Garrido; V. Martinez (2006). Changes in the contents of antioxidant compounds in pepper fruits at different ripening stages, as affected by salinity. Food Chem., 96: 66-73

Pablo, J. M. P.; M. S. William (2005). Effects of gibberellic acid, acetylthiproline and amixture of amino acids + short chain peptides on scallion yield. www. griffin. peachnet. Edupgrachrleston PGRSA - proceeding, 115-118.

Pelayo, C.; S. E. Ebeler; A. A. Kader (2003). Postharvest life and flavor quality of three strawberry cultivars kept at $5^{\circ} \mathrm{C}$ in air or air $+20 \mathrm{kPa} \mathrm{CO}$. PostharVest Biol. Technol., 27: 171-183.

Prasad, P. V. V. (2003). Iron Chlorosis. Nutrition, 649-656.
Rao, R. G. S.; P. M. Singh; M. Rai (2006). Storability of onion seeds and effects of packaging and storage conditions on viability and vigour. Scientia Horticulturae, No. 110: 1-6.

Rodrigues, A. S.; M. R. Pérez-Gregorio; M. S. García-Falcón; J. Simal-Gándara; D. P. F. Almeida (2010). Effect of post-harvest practices on flavonoid content of red and white onion cultivars. Food Control, Vol. 21: 878-884.

Sharma, M. K.; S. Karan; O. P. Gill (2004). Efficacy of dry permeation seed treatment with plant growth regulators in onion (Allium cepa L.) genotypes. Journal of Eco Physiology, 7(3/4): 101-104.

Sharma, R. C.; S. S. Gill; K. Neelu (2002). Pathological problems in production and storage of onion seed in Punjab and their remedial measures. Seed Research, 30(1): 134-141.

Siegenthaler, P. A.; O.V. Douet (1994). Relationship between the ATP content measured at three imbibition times and germination of onion seeds during storage at 3,15 and 30 degrees C. Journal of Experimental Botany, 45(279): 1365-1371.

Stanwood, P. C.; S. Sowa (1995). Evaluation of onion (Allium сера) seed after 10 years of storage at $5,-18$, and -196 degrees C. Crop Science, 35(3): 852-856.

Thomas, T. H.; F. M. R. Isenberg (1972). Hormone physiology of onion bulbs during dormancy. Exp. Hortic. 23: 48-51.

Uddin, M., H. S. MacTavish (2003). Controlled atmosphere and regular storage-induced changes in $S$-alk(en)yl-l-cysteine sulfoxides and alliinase activity in onion bulbs (Allium cepa L. cv. Hysam). Postharvest Biol. Technol. 28: 239-245.

Waller, R. A.; D. B. Duncan (1969). A bays rule for the symmetric multiple comparison problems. Amer. Stat. Assoc. J., 64: 14851503

Wood, M. (2008). The Earthwise Herbal: A complete guide to old world medicinal plants. north atlantic books. Berkeley, California, 69-70.

Xin, L.; Q. Wang (2006). The changes of the seed vigor of welsh onion under ultra-dry storage. China Vegetables. 8, 10-13. Cited from CAB Abstracts.

Yanping, Y.; G. Rongqi; S. Qingquan; L. Shengfu (1999). Effects of storage temperature and container type on the vigour of Welsh onion seeds with low moisture content. Australian Journal of Experimental Agriculture, 39(8): 1025-1028.

Yanping, Y.; G. Rongqi; S. Qingquan; L. Shengfu (2000). Vigour of welsh onion seeds in relation to storage temperature and seed moisture content. Seed Science and Technology, 28(3): 817-823.

Zhang, W. S., X. Li; J. T. Zheng; G. Y. Wang; C. De Sun; I. B. Ferguson, et al. (2008). Bioactive components and antioxidant capacity of Chinese bayberry (Myrica rubra Sieb and Zucc.) fruit in relation to fruit maturity and postharvest storage. European Food Research and Technology, 227, 1091-1097. 